\title{
Miscible displacement flows in near-horizontal ducts at low Atwood number
}

\author{
By S. M. TAGHAVI， K. ALB A ${ }^{2}$, T. SEO N ${ }^{3}$ \\ $\begin{array}{lll}\text { K. W I E L A G E-B U R C H A R D } & \text { D. M. M A R T I N E Z } \\ & 1\end{array}$ \\ AND I. A. F R I G A A R D $\mathbf{D}^{2,4} \dagger$
}

\footnotetext{
${ }^{1}$ Department of Chemical and Biological Engineering, University of British Columbia, 2360 East Mall, Vancouver, BC, V6T 1Z3, Canada.

${ }^{2}$ Department of Mechanical Engineering, University of British Columbia, 2054-6250 Applied Science Lane, Vancouver, BC, V6T 1Z4, Canada.

${ }^{3}$ Université Pierre et Marie Curie, Institut d'Alembert, 4 place Jussieu, 75005 Paris, France.

${ }^{4}$ Department of Mathematics, University of British Columbia, 1984 Mathematics Road, Vancouver, BC, V6T 1Z2, Canada.
}

(Received ?? and in revised form ??)

We study buoyant displacement flows with two miscible fluids of equal viscosity in the regime of low Atwood number and in ducts that are inclined close to horizontal. Using a combination of experimental, computational and analytical methods, we characterise the transitions in the flow regimes, between inertial and viscous dominated regimes, and as the displacement flow rate is gradually increased. Three dimensionless groups largely describe these flows: $\mathrm{Fr}$ (densimetric Froude number), Re (Reynolds number) and $\beta$ (duct inclination). Our results show that the flow regimes collapse into regions in a two-dimensional $(F r, R e \cos \beta / F r)$-plane. These regions are qualitatively similar between pipes and plane channels, although viscous effects are more extensive in pipes. In each regime we are able to give a leading order estimate for the velocity of the leading displacement front, which is effectively a measure of displacement efficiency.

\section{Introduction}

We consider high Péclet number miscible displacement flows in near-horizontal ducts (pipes and plane channels) with a heavier fluid displacing a lighter fluid downwards, i.e. density unstable. Such flows occur in many oil industry processes, concerned with either well construction (drilling, cementing, fracturing) or production (pipelining), as well as in other process industries. Laminar flows often occur in these processes, due to either high viscosities or other process constraints, and non-Newtonian fluids are also prevalent. In many situations it is not feasible to physically separate fluid stages as they are pumped. Two practical questions are A: to what degree does the fluid mix across the duct, $\mathbf{B}$ : what is the axial extent along the duct of the mixed region (meaning that in which we find both fluids present)? The aim of our paper is to present results of an extensive study, targeted primarily at understanding $\mathbf{B}$ in the case of long near-horizontal ducts. At these inclinations, transverse buoyancy forces act to stratify the fluid streams during the displacement flow, so that many flows are relatively laminar and structured. For other flows, inertial instabilities grow at the interface signifying the onset of mixing, which is often limited by buoyancy at these inclinations. In both cases $\mathbf{B}$ is answered by 
estimating the displacement front velocities. Only low Atwood numbers are considered $\left(A t=\left[\hat{\rho}_{H}-\hat{\rho}_{L}\right] /\left[\hat{\rho}_{H}+\hat{\rho}_{L}\right]\right)$ and the density of the displacing fluid $\left(\hat{\rho}_{H}\right)$ always exceeds that of the displaced fluid $\left(\hat{\rho}_{L}\right)$. Thus, buoyancy acts to spread the fluids along the duct and lengthen the mixed region. Only iso-viscous fluids are considered (viscosity $\hat{\mu}$ ).

Exchange flow in an inclined pipe (imposed mean flow velocity, $\hat{V}_{0}=0$ ) has been extensively studied by Seon et al. (2004, 2005, 2006, $2007 a, b)$, also considering iso-viscous fluids and low At. Seon et al. (2005) classify exchange flows phenomenologically as either inertial or viscous according to which effect is dominant in balancing buoyancy forces, and we adopt the same terminology. Where the flow remains primarily laminarised and uni-directional, with a clean interface and no evidence of instability, this is referred to as viscous. Where two and three-dimensional regions of flow are observed, typically associated with instability and (at least localised) mixing close to the interface, this is referred to as inertial. Seon et al. (2005) succeed in giving quantitative predictions of the front velocities at each inclination in terms of characteristic inertial and viscous velocity scales, $\hat{V}_{t}$ and $\hat{V}_{\nu}$ respectively:

$$
\hat{V}_{t}=\sqrt{\frac{\left[\hat{\rho}_{H}-\hat{\rho}_{L}\right] \hat{g} \hat{D}}{\left[\hat{\rho}_{H}+\hat{\rho}_{L}\right]}}=\sqrt{A t \hat{g} \hat{D}}, \quad \hat{V}_{\nu}=\frac{\left[\hat{\rho}_{H}-\hat{\rho}_{L}\right] \hat{g} \hat{D}^{2}}{2 \hat{\mu}}=\frac{A t \hat{\rho} \hat{g} \hat{D}^{2}}{\hat{\mu}},
$$

$\left(\hat{\rho}=\left[\hat{\rho}_{H}+\hat{\rho}_{L}\right] / 2, \hat{D}=\right.$ pipe diameter, $\hat{g}=$ gravitational acceleration $)$. Evidently $\hat{V}_{t}$ and $\hat{V}_{\nu}$ represent velocities at which buoyancy is balanced by inertial and viscous forces, respectively. The novelty of our work with respect to Seon et al. (2004, 2005, 2006, $2007 a, b)$ is the study of imposed displacement velocities, $\hat{V}_{0}>0$.

Preliminary results of our study were reported in Taghavi et al. (2010). As an increasingly strong mean flow, $\hat{V}_{0}>0$, was imposed on a pipe exchange flow the observations suggested a primary classification in terms of 3 regimes.

p1: Exchange flow dominated regime (low $\hat{V}_{0}$ ).

p2: Imposed flow dominated regime (moderate $\hat{V}_{0}$ ).

p3: Fully mixed regime (high $\hat{V}_{0}$ ).

Counter-intuitively, it was observed that increasing the imposed mean flow could even cause an inertial exchange flow to become progressively laminar and stable, $\mathbf{p} \mathbf{1} \rightarrow \mathbf{p} \mathbf{2}$, i.e. injecting inertia reduces instability. Taghavi et al. (2011) studied the flows that occur at the transition between regimes p1 \& p2. Right at the transition we find flows for which the less dense displaced fluid remains in a stationary layer at the top of the pipe, over the relatively long duration of our experiments, e.g. layers remained for $\hat{t} \gtrsim$ $10^{3} \hat{D} / \hat{V}_{0}$. Although the layer is stationary the fluid within the layer is in counter-current motion, with zero net flux. The same phenomenon was observed in channel displacement flows, simulated numerically. Good quantitative predictions of the stationary layer were obtained by analysing a lubrication/thin film model, extending from Seon et al. (2005); Taghavi et al. (2009).

Taghavi et al. (2011) studied flows at the transition $\mathbf{p} \mathbf{1} \rightarrow \mathbf{p} \mathbf{2}$, leading to a secondary classification based on the behaviour of the trailing front near the top of the pipe.

s1: Sustained back flows, buoyancy forces are strong enough to produce a sustained upstream motion of the trailing front (low $\hat{V}_{0}$ ).

s2': Stationary back flow, as described above, which is simply a marginal state.

s2: Temporary back flows, when the trailing front moved upstream only for a finite time, eventually reversing and moving downstream (low-moderate $\hat{V}_{0}$ ).

s3: Instantaneous displacements, when the trailing front moves directly downstream, (moderate-high $\hat{V}_{0}$ ).

The novelty of the work in this paper, compared to Taghavi et al. $(2010,2011)$ is 
threefold. First, the results of Taghavi et al. (2010) were preliminary, based only on a limited number of pipe flow experiments. In this paper we confirm the generality of these results, both with a much larger data set and with a second geometry (plane channel). We also characterise the dynamics of each regime in significantly more detail than possible in Taghavi et al. (2010). In particular we show via a similarity scaling that regime p2 is a viscous regime. Secondly, although Taghavi et al. (2011) studied the transition $\mathbf{p} \mathbf{1} \rightarrow \mathbf{p} \mathbf{2}$, it is focused on the stationary back flows $\mathbf{s} \mathbf{2}$ '. This leaves largely unexplored the relation between the primary and secondary classifications. Also missing are quantitative estimates of the front velocity, $\hat{V}_{f}$, which are essential for predicting displacement efficiencies. Essentially the displacement efficiency is proportional to $\hat{V}_{0} / \hat{V}_{f}$, as discussed in Taghavi et al. (2009). From a more fundamental perspective, both primary and secondary classifications are phenomenological. They describe displacement front behaviour but do not classify the physics, e.g. whether a particular regime is stable/unstable or viscous/inertial. Here we develop and present a complete classification of these flows that includes leading order predictions of front velocity in each regime and a description of the primary physical balances. This is done for both pipe and channel geometries. Thirdly later in the paper, we consider the issue of how to model inertial effects for flows with greatly elongated displacement fronts (as we observe). We adopt the weighted-residual approach for two-layer flows; see ?, which allows us to consider weak inertial effects on both the flow stability (long wavelengths) and on the displacement front shape and velocity.

Regarding the existing literature, high Péclet number miscible displacements have been studied analytically, computationally, and experimentally in the case of iso-dense fluids, by Petitjeans \& Maxworthy (1996); Chen \& Meiburg (1996); Rakotomalala et al. (1997); Yang \& Yortsos (1997). These studies show that sharp interfaces persist over wide ranges of parameters for dimensionless times (hence lengths) smaller than the Péclet number. At longer times (lengths) the dispersive limit is attained. However, for fixed lengths and for increasing Péclet number (while remaining laminar) the flow is comparable to an immiscible displacement (with zero-surface tension). On including buoyancy, there are a number of displacement flow studies in vertical ducts, both for miscible and immiscible fluids, e.g. Joseph \& Renardy (1993); Lajeunesse et al. (1997, 1999); Sahu et al. (2009b).

When buoyancy forces are strong the flow characteristics can vary considerably with inclination. The most comprehensive study of these effects is in the absence of the imposed flow, by Seon et al. $(2004,2005,2006,2007 b, a)$ using experimental methods. Three-dimensional computations have been performed by Hallez \& Magnaudet (2008) and largely confirm the qualitative picture of Seon et al. $(2005,2006)$. The computations of Hallez \& Magnaudet (2008) are focused mostly at inclinations away from horizontal and they consider mostly inertial flows. They also study exchange flows in plane channels, concluding that the structure of buoyancy-inertia-driven flows is markedly different in $2 \mathrm{D}$ and $3 \mathrm{D}$ settings. This is largely due to the $3 \mathrm{D}$ effects of mixing. In vertical pipes there have been a range of exchange flow studies focused towards the study of mixing in inertia-buoyancy dominated regimes, e.g. Baird et al. (1992); Debacq et al. (2001, 2003), but also more geophysically oriented studies at low Reynolds number, e.g. Stevenson \& Blake (1998); Huppert \& Hallworth (2007); Beckett et al. (2011). There are also many general studies of buoyancy-driven flows of miscible Newtonian fluids over near-horizontal surfaces in oceanographic, meteorological and geophysical contexts, e.g. Benjamin (1968); Hoult (1972); Didden \& Maxworthy (1982); Simpson (1997); Shin et al. (2004); Birman et al. $(2005,2007)$.

Much of our study concerns regimes for front propagation. However, in our results 


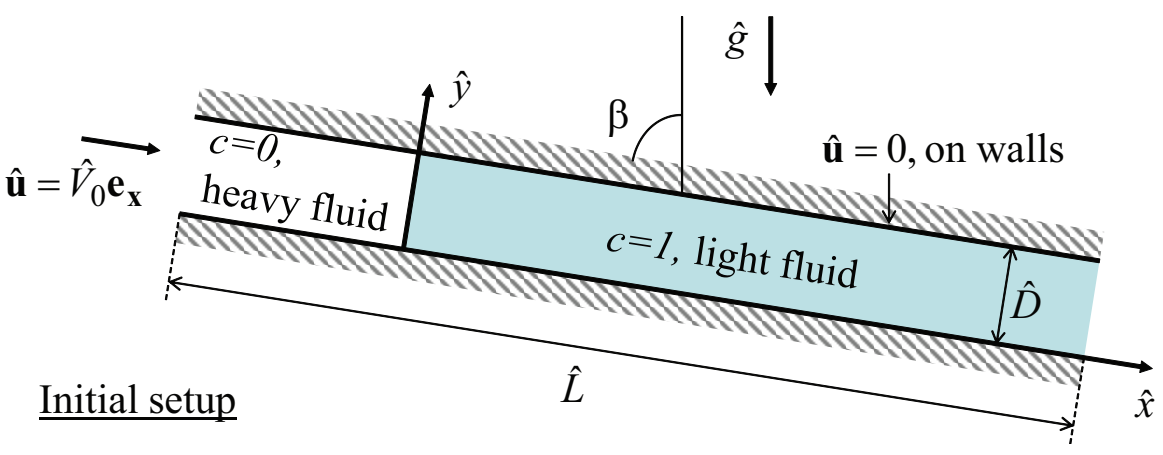

FiguRE 1. Schematic of the flows studied.

we also observe instabilities at the interface as it elongates. There are a limited number of studies associated with instability of such flows, e.g. Goyal \& Meiburg (2006); Goyal et al. (2007) and Sahu et al. $(2009 b, a)$. Once the interface elongates, the flow on any particular cross-section is not distinguishable from a miscible multi-layer flow. There is also an extensive literature on the instability of immiscible parallel multi-layer flows, dating from the classical study of Yih (1967). Explanations of the physical mechanisms that govern this type of instability for Newtonian fluids have been offered by Hinch (1984); Charru \& Hinch (2000). In the context of miscible multi-fluid flows there is less work on shear instabilities, where the term multi-layer is ill-defined if the fluids can mix. Linear stability studies assume a quasi-steady parallel base state. Ranganathan \& Govindarajan (2001) and Govindarajan (2004) analysed the stability of miscible fluids of different viscosities flowing through a channel in a three-layer Poiseuille configuration. They obtained instabilities at high Schmidt numbers and low Reynolds numbers, resembling those of Yih (1967). In Couette flow it appears that the stability characteristics of the miscible flow are predicted by those of the immiscible flow with zero surface tension; see Ern et al. (2003). However, for core annular flow this is not the case; see Selvam et al. (2007). Recent studies have considered convective instabilities in miscible multi-layer flows, both experimentally by d'Olce (2008); d'Olce et al. $(2008,2009)$ and computationally/analytically by Selvam et al. (2009). Sahu et al. (2009b,a) have recently considered the onset of convective instabilities in 3-layer plane channel flows.

\subsection{Problem Setting}

The scenario studied throughout the paper is that fluid 1 displaces fluid 2 along a duct that is inclined close to horizontal. The duct has transverse dimension $\hat{D}$ (either the pipe diameter or the channel height), and the mean imposed displacement velocity is $\hat{V}_{0}$, in the downhill direction. The fluids have the same viscosity $\hat{\mu}$, are miscible and have differing densities: the displacing fluid 1 is heavier than the displaced fluid 2. In general we study laminar flows. The length $\hat{L}$ of the duct satisfies $\hat{L} \gg \hat{D}$ and the initial interface is transverse to the duct axis, somewhere away from the ends; see Fig. 1.

From a modeling perspective a natural formulation involves a concentration-diffusion equation coupled to the Navier-Stokes equations. The change between pure fluids 1 and 2 is modeled via a scalar concentration, $c$. On making the Navier-Stokes equations dimensionless using $\hat{D}$ as length-scale, $\hat{V}_{0}$ as velocity scale, and subtracting a mean static pressure gradient before scaling the reduced pressure, we arrive at:

$$
[1+\phi A t]\left[\mathbf{u}_{t}+\mathbf{u} \cdot \nabla \mathbf{u}\right]=-\nabla p+\frac{1}{R e} \nabla^{2} \mathbf{u}+\frac{\phi}{F r^{2}} \mathbf{e}_{g}
$$




$$
\begin{aligned}
\nabla \cdot \mathbf{u} & =0, \\
c_{t}+\mathbf{u} \cdot \nabla c & =\frac{1}{P e} \nabla^{2} c .
\end{aligned}
$$

Here $\mathbf{e}_{g}=(\cos \beta,-\sin \beta)$ and the function $\phi(c)=1-2 c$ interpolates linearly between 1 and -1 for $c \in[0,1]$. The 4 dimensionless parameters appearing in (1.2) are the angle of inclination from vertical, $\beta$, the Atwood number, $A t$, the Reynolds number, $R e$, and the (densimetric) Froude number, Fr. The last two are defined as:

$$
R e \equiv \frac{\hat{V}_{0} \hat{D}}{\hat{\nu}}=\frac{\hat{V}_{0} \hat{V}_{\nu}}{\hat{V}_{t}^{2}}, \quad F r \equiv \frac{\hat{V}_{0}}{\sqrt{A t \hat{g} \hat{D}}}=\frac{\hat{V}_{0}}{\hat{V}_{t}} .
$$

Here $\hat{\nu}$ is defined using the mean density $\hat{\rho}$ and the common viscosity $\hat{\mu}$ of the fluids.

Also in (1.4) appears a 5th dimensionless group, the Péclet number, $P e=\hat{V}_{0} \hat{D} / \hat{D}_{m}$, with $\hat{D}_{m}$ the molecular diffusivity (assumed constant for simplicity). Commonly the Péclet number is very large as we consider lab/industrial scale flows rather than microfluidic devices, e.g. $\mathrm{Pe}>10^{6}$ is common. If the fluids are initially separated we expect diffusive effects to be initially limited to thin interfacial layers of size $\sim P e^{-1 / 2}$, remaining sharp over experimental timescales in the absence of instability, mixing and dispersion. Such flows are close to their immiscible fluid analogues at infinite capillary number (i.e. vanishing surface tension) which are modeled by setting $P e=\infty$ and ignoring the right-hand-side of (1.4). The direct effect of the density difference on inertia is captured by At. If we restrict our attention to density differences of the order of $10 \%$ (as in our experiments) we see that $A t \leqslant 0.05$. We expect that for such moderate density differences the solution for $A t=0$ will give a reasonable approximation. Note also that the incompressibility condition (1.3) in fact requires small $A t$ in order to be valid for intermediate $c$ in the case that the 2 individual pure fluids can be considered incompressible. Essentially this is the Boussinesq approximation. Note that for such flows we can still have significant buoyancy effects (captured by $R e / F r^{2}$ ). Thus, the overall aim of our study is to build a quantitative description of the different flow regimes found, in terms of $(R e, F r)$, for $\beta$ close to $\pi / 2$, assuming $P e \rightarrow \infty$ and $A t \rightarrow 0$.

\subsection{Outline}

The main content of our paper proceeds in 3 sections. The first section $(\S 2)$ concerns pipe flow displacements. The main methods are experimental and semi-analytical, using a lubrication/thin-film modeling approach. The second section (§3) presents analogous studies in a plane channel geometry. Here the physical experiments are replaced with numerical experiments. In both geometries we obtain reasonable agreement with predictions from the semi-analytical models. The discrepancies are possibly attributable to inertial effects, which we study in $\S 4$. We also study the flow stability in $\S 4$. The paper ends with a brief summary.

\section{Displacement in pipes}

\subsection{Experimental description}

Our experimental study was performed in a 4 (m) long, 19.05 (mm) diameter, transparent pipe with a gate valve located $80(\mathrm{~cm})$ from one end. A schematic of the experimental set-up is given in Fig. 2 and a detailed description can be found in Taghavi et al. (2010, 2011). The pipe was mounted on a frame which could be tilted to a given angle. Initially, the lower part of the pipe was filled with a lighter fluid coloured with a small amount 


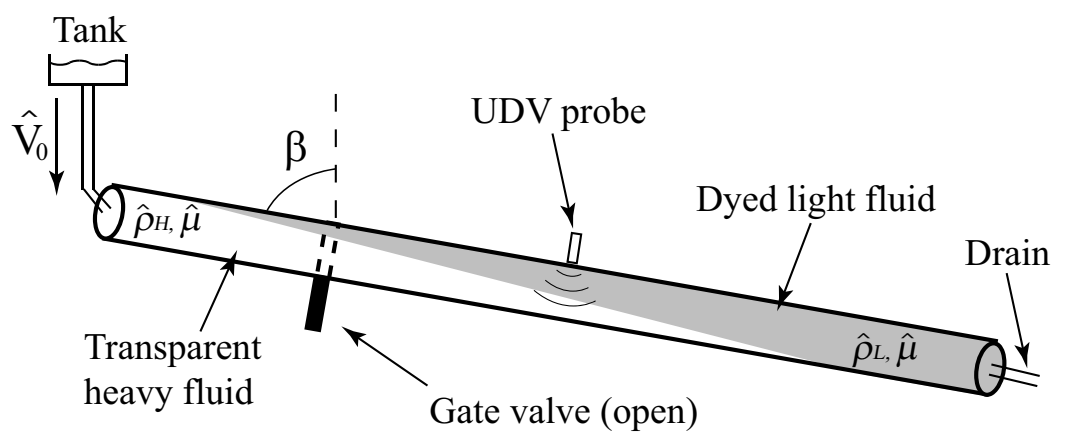

Figure 2. Schematic view of experimental set-up. The interface shape is illustrative only.

\begin{tabular}{c|ccc||cc}
$\beta^{\circ}$ & $\hat{\nu}\left(\mathrm{mm}^{2} \cdot \mathrm{s}^{-1}\right)$ & $A t\left(\times 10^{-3}\right)$ & $\hat{V}_{0}\left(\mathrm{~mm} \cdot \mathrm{s}^{-1}\right)$ & $R e$ & $F r$ \\
\hline $83 \dagger$ & $1-2$ & $1-40$ & $0-841$ & $0-16021$ & $0-19.45$ \\
85 & $1-2$ & $1-91$ & $0-80$ & $0-1524$ & $0-5.37$ \\
87 & $1-2$ & $1-10$ & $0-77$ & $0-1467$ & $0-5.63$
\end{tabular}

$\dagger$ Most of the experiments were conducted in the ranges At $\left(\times 10^{-3}\right) \in[1,10], \hat{V}_{0} \in 0-110\left(\mathrm{~mm} . \mathrm{s}^{-1}\right)$.

TABLE 1. Experimental plan.

of ink, and the upper part by a denser solution. The pipe was fed from an elevated tank, forced by gravity to avoid disturbances induced by a pump. The imposed flow rate was controlled by a valve and measured by both a rotameter and a magnetic flowmeter, located downstream of the pipe. We also measured the velocity profile at $80(\mathrm{~cm})$ below the gate valve, using an ultrasonic Doppler velocimeter DOP2000 (model 2125, Signal Processing SA). Most of the experiments were conducted using water as the common fluid, with salt $(\mathrm{NaCl})$ as a weighting agent to densify one of the fluids. To achieve higher viscosity, glycerol solutions were prepared by diluting pure glycerol with water. Our experiments were conducted over the ranges shown in Table 1.

Our main measurement method was based on quantitative image analysis, extracting information regarding large-scale features of the flow such as the front velocity. The imaging system consisted of 2 digital cameras with images recorded at a frame rate of typically 2 or $4(\mathrm{~Hz})$. Each of these cameras covered $160(\mathrm{~cm})$ of the lower part of the pipe. In order to help the visualization of the phases, the pipe was illuminated from behind by a light box containing fluorescent light tubes filtered through a diffusive paper giving a homogeneous light. Light absorption calibration was carried out for both cameras. During the experiment (after opening the gate valve), images were obtained at regular time intervals, which enabled us to create spatiotemporal diagrams of the averaged concentration profiles along the length of the pipe. The fronts were marked on these diagrams by a sharp boundary between the different relative concentrations of the fluids. The front velocities were obtained from the slope of this boundary. Examples of this analysis can be found in Taghavi et al. (2010, 2011).

We first calibrated our apparatus against exchange flow results of Seon et al. (2005, 


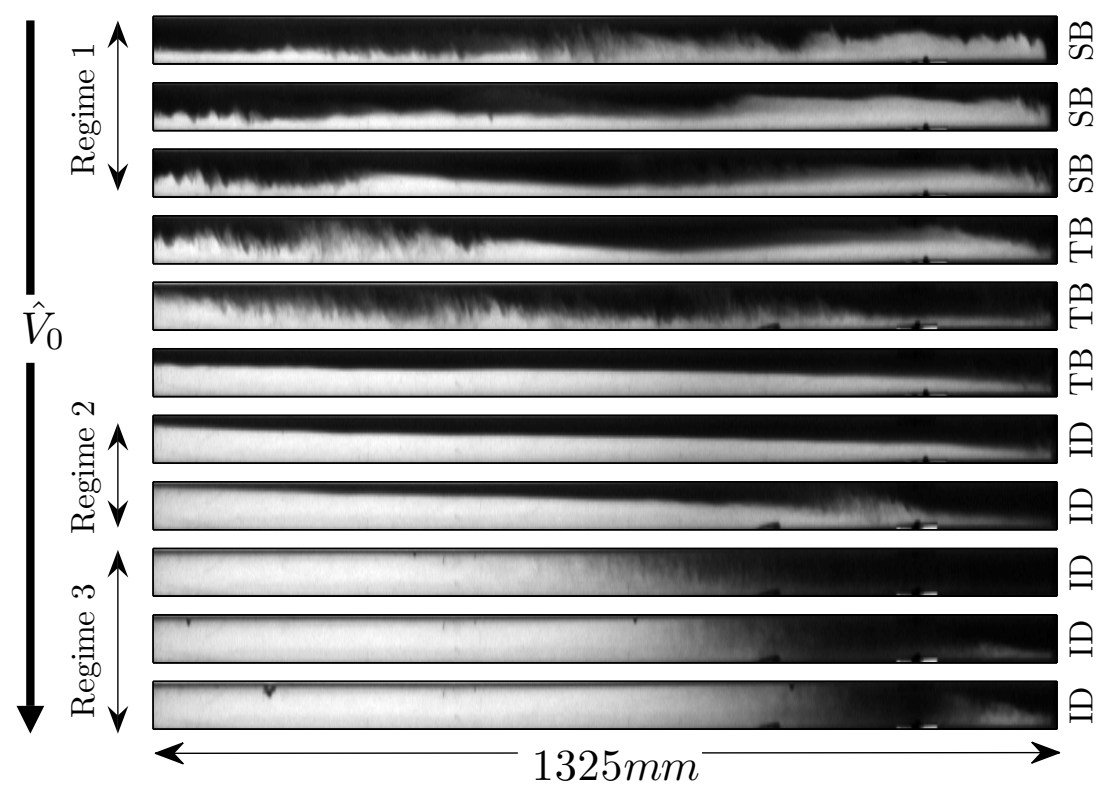

Figure 3. A sequence of snapshots from experiments with increased imposed flow rate; $\beta=83^{\circ}, A t=10^{-2}$ and $\hat{\nu}=1\left(\mathrm{~mm}^{2} \cdot \mathrm{s}^{-1}\right)$. From top to bottom we show images for

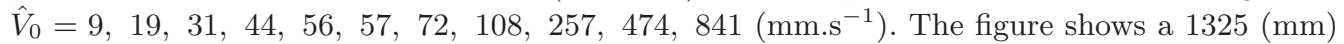
long section of the pipe a few centimeters below the gate valve. Regimes 1-3 are the primary classification p1-p3 in $\S 1$. The secondary classification (s1-s3) is also made: SB = sustained backflow; $\mathrm{TB}=$ temporary backflow; ID = instantaneous displacement.

$2007 b$ ) for different Atwood numbers at $\beta=85^{\circ}$ and $\beta=87^{\circ}$. The errors in measured front velocity were always below $2 \%$ for the cases studied and the experiments had a high degree of repeatability.

\subsection{Observations}

In a typical displacement experiment we observe a short inertial phase following the opening of the gate valve. The fluids are initially at rest. When the gate valve is opened the static head accelerates both fluids from rest and at the same time the density difference between fluids accelerates the fluids in opposing directions. This first stage is very fast (order of seconds). Inertia is always the main balancing force for buoyancy in the first part of the experiment, when the interface is transverse to the pipe axis. Although the time evolution from an initial acceleration phase to an inertia-buoyancy balance (and potentially thereafter to a viscous-buoyancy balance) is interesting in itself, for most of our study we disregard the initial phase and concentrate on characterizing longer time dynamics.

Over longer times we characteristically observe two fronts emerging. The leading front is towards the bottom of the pipe and moves downstream faster than the mean flow. The trailing front is towards the top of the pipe and moves slower than the leading front. Depending on the buoyancy forces the trailing front may move either upstream against the mean flow (buoyancy forces dominate imposed flow) or downstream (imposed flow dominates buoyancy forces). The front may also move initially upstream and then become washed downstream over a longer time interval. The interface between these two advancing fronts is essentially stretched axially along the pipe. These regimes correspond 

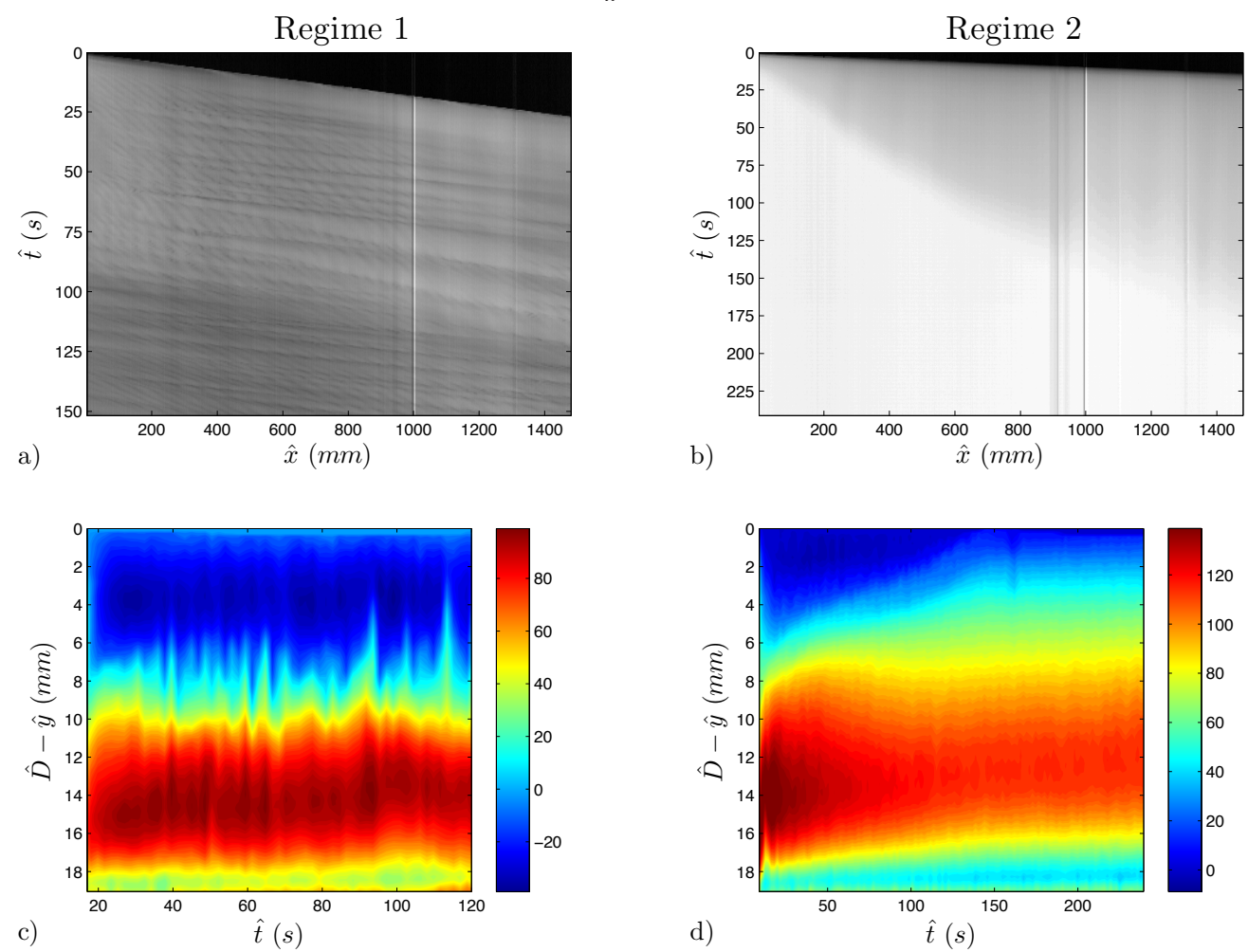

FiguRE 4. Examples of spatiotemporal diagrams and corresponding UDV measurements obtained for $\beta=85^{\circ}, A t=10^{-2}$ and $\hat{\nu}=1\left(\mathrm{~mm}^{2} \cdot \mathrm{s}^{-1}\right)$ : a) \& c) $\hat{V}_{0}=30\left(\mathrm{~mm} \cdot \mathrm{s}^{-1}\right)$; b) \& d) $\hat{V}_{0}=75\left(\mathrm{~mm} \cdot \mathrm{s}^{-1}\right)$. The velocity is measured through the pipe centreline in a vertical section, with the UDV angled at $74^{\circ}$ to the surface of the pipe, positioned at $80(\mathrm{~cm})$ below the gate valve. The vertical axis shows depth measured from the top of the pipe. Velocity contours are averaged in time over 15 consecutive velocity profiles, $(1.8(\mathrm{~s}))$.

to the secondary classification s1-s3 described in $\S 1$, and have been explained in Taghavi et al. (2011). Here we are more interested in the primary classification p1-p3, observed by Taghavi et al. (2010) as $\hat{V}_{0}$ increases. Certainly one of the most interesting aspects of the transition between regimes is the laminarisation as $\hat{V}_{0}$ increases. We take a more detailed look at this transition here.

In Fig. 3 we show snapshots from a sequence of experiments performed for progressively large $\hat{V}_{0}$. In this case the pure exchange flow $\left(\hat{V}_{0}=0\right)$ is strongly inertial and in the first few snapshots we see a propagating layer of heavy fluid at the bottom of the pipe with a significant mixed layer on top. At intermediate imposed velocities we see the clear laminarisation of the flow (e.g. at $\left.\hat{V}_{0}=57 \& 72\left(\mathrm{~mm} . \mathrm{s}^{-1}\right)\right)$. Finally at larger $\hat{V}_{0}$ we see progressively more mixing, except now there is sufficient inertia to mix across the whole pipe cross-section. The secondary classification (s1-s3) is also shown in Fig. 3.

Examples of spatiotemporal diagrams related to flows in the first and second regimes (p1 \& p2) are shown in Fig. 4a-b, (from a different sequence than Fig. 3). For the parameters selected, the pure exchange flow is again inertial. For low $\hat{V}_{0}=30\left(\mathrm{~mm} . \mathrm{s}^{-1}\right)$, the flow remains unstable. In Fig. 4a we can observe the initial front propagating and behind it unstable waves appear at the interface, as evidenced below the initial sharply defined dark region in Fig. 4a. We observe a range of wave speeds differing slightly from the front propagation speed. No second front is observed, as for this experiment 

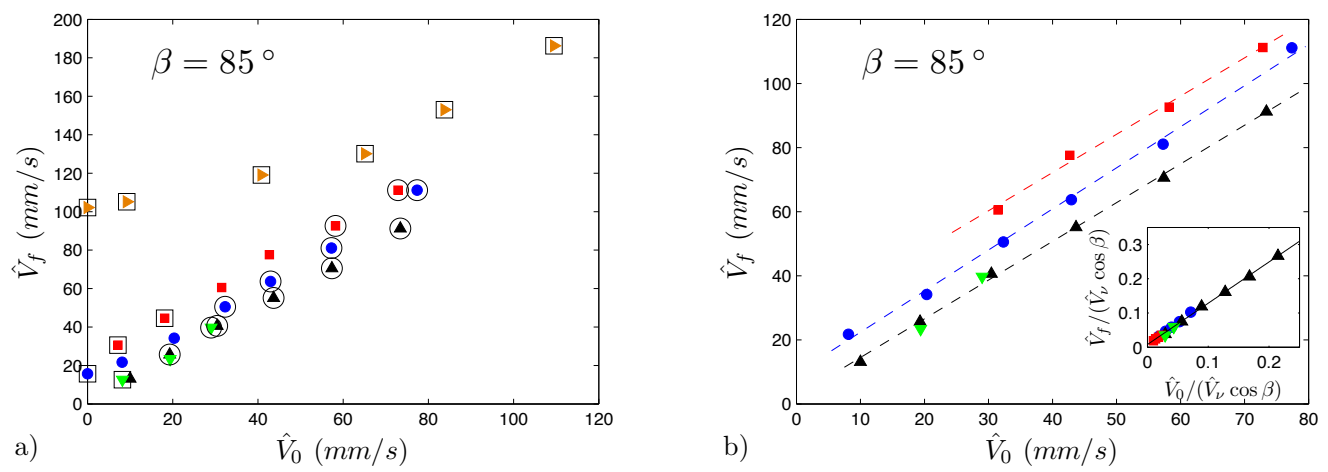

FiguRE 5 . Variation of the front velocity $\hat{V}_{f}$ as a function of mean flow velocity $\hat{V}_{0}$ for different values of density contrast and viscosity at $\beta=85^{\circ}$. a) Sustained back flows (s1) and instantaneous displacements (s3) are marked by the superposed squares and circles, respectively. Data points without marks are either temporary back flows, stationary interfaces or undetermined experiments. b) Illustration of the imposed flow dominated regime (p2), compared to the left plot all sustained back flows are excluded. The dashed lines are linear fits of data points for each set of increasing $\hat{V}_{0}$ (fixed $A t$ and $\hat{\nu}$ ). The inset shows normalized front velocity $\hat{V}_{f} / \hat{V}_{\nu} \cos \beta$ as a function of normalized mean flow velocity $\hat{V}_{0} / \hat{V}_{\nu} \cos \beta$, for which the data superimpose. The solid line is a linear fit to all the normalized data points. In both figures the data correspond


$\nu=1\left(\mathrm{~mm}^{2} \cdot \mathrm{s}^{-1}\right)$ and $A t=3.7 \times 10^{-3}(\nabla)$ with $\hat{\nu}=1.7\left(\mathrm{~mm}^{2} \cdot \mathrm{s}^{-1}\right)$.

the trailing front moves backward, upstream against the flow. For an increased $\hat{V}_{0}=$ 75 (mm.s $\left.{ }^{-1}\right)$ the flow has become stable; see Fig. $4 \mathrm{~b}$. The slope of the line separating the black region and gray region represents the velocity of the leading front, at the lower wall. We can also discern a separating curve between the gray and white regions: the slope of this curve represents the (lower) velocity of the trailing front at the upper wall.

The corresponding UDV results for the same two experiments are shown in Fig. 4c-d. In Fig. 4c we observe temporal oscillations corresponding to the flow instability. The sustained back flow is evident in the negative velocity values at the top of the pipe. The stable flow is illustrated in Fig. 4d. The UDV images are ensemble averaged over 15 consecutive images, corresponding to a time average over a local interval of 1.8 (s). This eliminates small high frequency fluctuations, which correspond to the UDV sampling rate. If we look carefully, we can observe the presence of negative values of flow velocity towards the top of the tube. In this experiment there is no back flow of the trailing front, but this does not preclude negative velocities. These regions correspond to a temporary recirculation at this position inside the upper fluid, which persists for $\hat{t} \approx 125$ (s), by which time the trailing front reaches the UDV probe located at $\hat{x}=80(\mathrm{~cm})$. After the trailing front has passed, a more Poiseuille-like flow is recovered. Note also that in this initial period, when negative velocities are found in the upper layer, the velocities in the lower layer must be correspondingly higher (observe the dark red region) to maintain the fixed imposed flow rate.

Figures $3 \& 4$ capture many of the features of flows in regimes $\mathbf{p} 1 \& \mathbf{p} \mathbf{2}$, adding significantly more detail to the characterisation from Taghavi et al. (2010) which is based simply on the leading front velocity. It is also possible to have flows in regime $\mathbf{p} \mathbf{1}$ that are viscous (see later). The variation of leading front velocity with mean flow velocity $\hat{V}_{0}$ for all our experiments is qualitatively similar to that already observed in Taghavi et al. 
(2010). Typical results are plotted in Fig. 5a for different experimental sequences at fixed angle, $\beta=85^{\circ}$. In this figure we observe mostly regimes $\mathbf{p} 1 \& \mathbf{p} 2$ of the displacement, i.e. an initial plateau at low $\hat{V}_{0}$ (exchange flow regime) followed by linear increase in $\hat{V}_{f}$ at larger $\hat{V}_{0}$. The secondary classification (s1-s2) is also represented in Fig. 5a. As well as the transition from exchange flow dominated to imposed flow dominated displacement regimes, we also observe the transition from sustained back flow through to instantaneous displacement on each data set, as $\hat{V}_{0}$ is increased.

Figure 5b examines the imposed flow dominated regime $\mathbf{p} 2$ more closely for the data at inclination angle $\beta=85^{\circ}$. We exclude only those data points classified as sustained backflow (s2) and observe that for this case these correspond well to the imposed flow dominated regime and show an approximately linear increase with $\hat{V}_{0}$. The dashed lines give an approximate linear fit to each data set. The inset of Fig. 5b shows that by normalizing with $\hat{V}_{\nu} \cos \beta$ the data in the imposed flow dominated regime collapses onto a single curve, which we explain below in $\S 2.3$. We have found a similar data collapse with other experimental sequences in regime p2. It is this collapse of the data onto a single curve that establishes the essentially viscous nature of the flow in this regime.

\subsection{Lubrication/thin film model}

To explain the similarity scaling evident in our data (e.g. Fig. 5b), we resort to a lubrication/thin film style of model (assuming the immiscible limit $P e \rightarrow \infty$ ). This type of model has been developed for plane channel displacements in Taghavi et al. (2009). Exchange flows have been studied using this type of model in Seon et al. (2007b) and in Taghavi et al. (2011) we have extended this type of model to the displacement regimes studied here. For brevity, we refer to Taghavi et al. (2011) for the derivation.

The interface height evolution is governed by the following dimensionless equation:

$$
\frac{\partial}{\partial T} \alpha(h)+\frac{\partial}{\partial \xi} q\left(h, h_{\xi}\right)=0 .
$$

In this model $h \in[0,1]$ is the dimensionless interface height (scaled with the diameter), $\alpha(h) \in[0,1]$ is the area fraction occupied by the heavy fluid (under the interface)

$$
\alpha(h)=\frac{1}{\pi} \cos ^{-1}(1-2 h)-\frac{2}{\pi}(1-2 \mathrm{~h}) \sqrt{h-h^{2}}
$$

and the scaled flux of fluid in the heavy layer is denoted $q\left(h, h_{\xi}\right)$ :

$$
q\left(h, h_{\xi}\right)=\frac{32}{\pi} \int_{\alpha(h)}\left(\frac{1}{4}-x^{2}-y^{2}\right) d x d y+\frac{F_{0}\left[\chi-h_{\xi}\right]}{4}\left(1-(1-2 h)^{2}\right)^{7 / 2} .
$$

The first term is the Poiseuille component and the second term is the exchange flow component; $F_{0}$ is given by Seon et al. (2005) as $F_{0}=0.0118$. The variables $T$ and $\xi$ are the dimensionless time and length variables, respectively:

$$
T=\frac{\hat{t} \hat{V}_{0}}{\hat{D}} \delta, \quad \xi=\frac{\hat{x}}{\hat{D}} \delta
$$

where

$$
\delta=\frac{\hat{\mu} \hat{V}_{0}}{\left[\hat{\rho}_{H}-\hat{\rho}_{L}\right] \hat{g} \sin \hat{\beta} \hat{D}^{2}}=\frac{\hat{V}_{0}}{2 \hat{V}_{\nu} \sin \beta} .
$$

This type of model contains the balance between viscous, buoyant and imposed flow 
stresses. Only a single dimensionless parameter $\chi$ remains following the model reduction:

$$
\chi=\frac{\cot \beta}{\delta}=\frac{\left[\hat{\rho}_{H}-\hat{\rho}_{L}\right] \hat{g} \cos \hat{\beta} \hat{D}^{2}}{\hat{\mu} \hat{V}_{0}}=\frac{2 \hat{V}_{\nu} \cos \beta}{\hat{V}_{0}}=\frac{2 R e \cos \beta}{F r^{2}},
$$

which represents the balance of axial buoyancy stresses and viscous stresses due to the imposed flow. The interface slope $h_{\xi}$ generates additional axial pressure gradients which contribute to the exchange flow component of flux in (2.3), but as the interface extends progressively longer, this effect becomes irrelevant, except possibly in local regions. Thus, purely from the perspective of dimensional analysis, the similarity scaling evident in Fig. 5b (inset) is expected: it simply shows that the long-time front velocity depends uniquely on the parameter $\chi$.

Although the algebraic form of (2.1) differs from that analysed for the plane channel, we find qualitatively similar behaviour. Typically we find a short initial transient during which the interface elongates from its initial position and diffusive spreading due to the presence of the term $h_{\xi}$ in $q$ dominates the behaviour. This is followed by the emergence of a distinct leading front, which abuts the lower wall of the pipe (including $h=0$ ), and always propagates downstream at a speed $V_{f}>1$. By front we mean an interval of $h$ that moves at constant speed. At large values of $\chi$, buoyancy is strong and a second trailing front emerges that moves upstream. As $\chi$ is reduced the trailing front speed decreases until there is no back flow (at a critical $\chi=\chi_{c} \approx 116.32$ ). The interface displaces only in the positive direction for $\chi<\chi_{c}$.

The long time behaviour of the system is governed by the hyperbolic part of (2.1), i.e. setting $q=q(h, 0)$. The equations determining the leading front speed $\left(V_{f}\right)$ and front height $\left(h_{f}\right)$ are:

$$
\alpha\left(h_{f}\right) V_{f}=q\left(h_{f}, 0\right), \quad V_{f}=\frac{\partial q}{\partial h}\left(h_{f}, 0\right)\left[\frac{d \alpha}{d h}\left(h_{f}\right)\right]^{-1},
$$

which can be solved numerically to give $V_{f}(\chi)$.

The superposition of the experimental data shown in the inset of Fig. $5 \mathrm{~b}$ corresponds to a (near linear) variation of the normalized leading front velocity with $\chi^{-1}$. It is natural to compare the experimental front speeds with the calculated front speeds from our lubrication model. This is done in Fig. 6 for the full range of experimental data that fall in either exchange dominated regime or imposed flow dominated regimes (p1 \& p2). The bold line indicates the scaled front velocity obtained by the lubrication model, i.e. solving (2.7). The circle on the bold line indicates the theoretical balance between these two regimes, at $\chi=\chi_{c} \approx 116.32$ where the stationary layer flow (s2') is found for the lubrication model.

For values of $\chi<\chi_{c}$, where instantaneous displacements in the imposed flow dominated regime are found, the collapse of the data onto the theoretical curve is evident. This emphasizes that in this regime the balance is primarily between viscous forces generated by the imposed flow and buoyancy. Although we have a high degree of agreement with this simple model (considering also the experimental uncertainty), we note that the experimental data does generally lie just above the theoretical curve, in the imposed flow dominated regime. We hypothesize that this discrepancy is an effect of inertia and examine this possibility in $\S 4$.

\subsection{The exchange-flow dominated range}

In the region denoted by the circled 2 in Fig. 6 we see some experimental sequences that agree reasonably well with the viscous lubrication model, but also others that diverge 


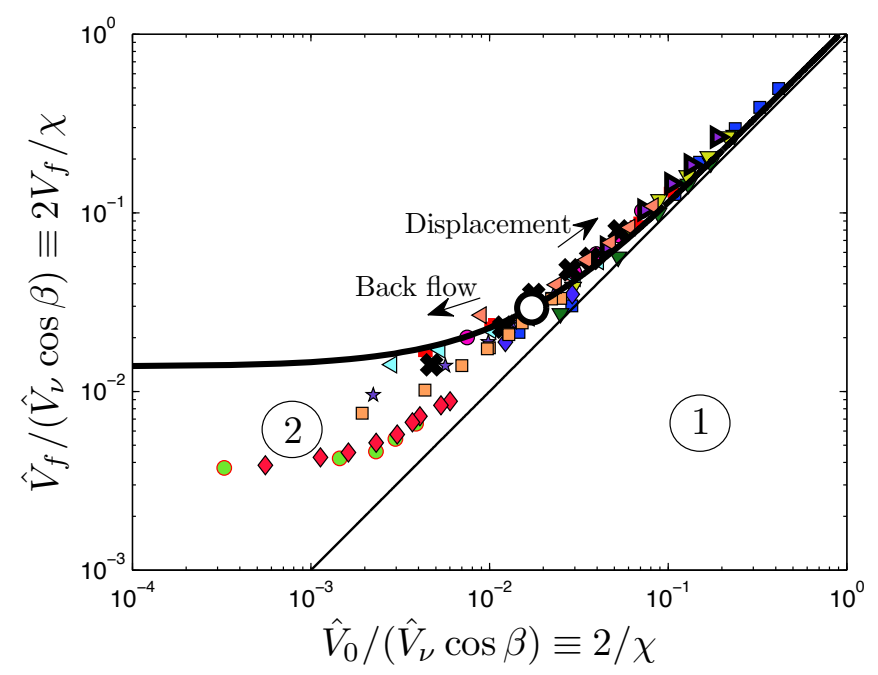

FiguRE 6. Normalized front velocity, $\hat{V}_{f} / \hat{V}_{\nu} \cos \beta$, plotted against normalized mean flow velocity, $\hat{V}_{0} / \hat{V}_{\nu} \cos \beta$, for the full range of experiments in the first and second regimes (limited by $R e<2300)$ in Table 1. Data points with the same symbols belong to experimental sets of increasing Reynolds number (via $\hat{V}_{0}$ ) for fixed $A t$ or viscosity. The heavy solid line indicates the scaled front velocity from the lubrication model. The circle indicates the theoretical transition $\left(\chi=\chi_{c} \approx 116.32\right)$. The thin solid line shows $\hat{V}_{f}=\hat{V}_{0}$, below which front velocities are not possible (denoted by the circled 1). The region denoted by the circled 2 represents flows with increasingly significant buoyant effects.

significantly. Inertial effects are not included in the lubrication approximation and thus divergence of the data from (2.7) suggests the increasing importance of inertia in in balancing the buoyancy-driven exchange component. It would be beneficial to have a predictive model also for this data, of similar accuracy to (2.7).

According to Seon et al. (2007b) inertial exchange flows in near-horizontal pipes are found for

$$
R e_{t} \cos \beta=\frac{\hat{V}_{\nu} \cos \beta}{\hat{V}_{t}} \gtrsim 50,
$$

and the front velocity can be approximated by

$$
\hat{V}_{f} \approx \gamma \hat{V}_{t}, \quad \text { with } \gamma \approx 0.7 \text {. }
$$

This scaling confirms that $\hat{V}_{t}$ is the relevant scale as $\hat{V}_{0} \rightarrow 0$ for inertially dominated flows. We might then consider that $\gamma$ is simply the leading order term in an expansion with respect to small $F r=\hat{V}_{0} / \hat{V}_{t}$, i.e. for $\chi \gg \chi_{c}$ we assume

$$
\frac{\hat{V}_{f}}{\hat{V}_{t}}=f(F r) \approx f(0)+F r f^{\prime}(0)+\frac{F r^{2}}{2} f^{\prime \prime}(0)+\ldots .
$$

where $f(0)=\gamma \approx 0.7$. With this ansatz we rescale $\hat{V}_{f}$ with $\hat{V}_{t}$ for all our experimental data $\chi>\chi_{c}$ that satisfies (2.8). We use this data to fit the coefficients in (2.9). We find $f^{\prime}(0)=0.595$ and $f^{\prime \prime}(0)=0.724$, which are in the confidence intervals $f^{\prime}(0) \in$ $(0.454,0.735)$ and $f^{\prime \prime}(0) \in(0.478,0.970)$ with confidence level $95 \%$. Figure 7a shows a 

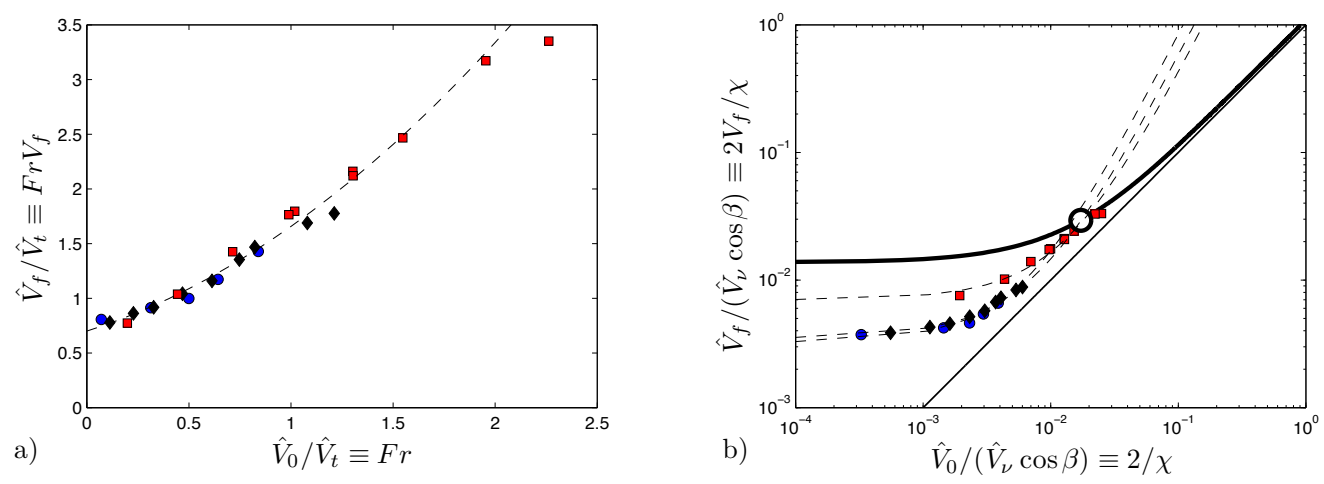

FiguRE 7. a) Normalized front velocity, $\hat{V}_{f} / \hat{V}_{t}$, as a function of normalized mean flow velocity $\hat{V}_{0} / \hat{V}_{t}=F r$, (equivalently Froude number), plotted for 3 experimental sequences in the inertial regime. Data correspond to $A t=9.1 \times 10^{-2}$ at $\beta=85^{\circ}(\bullet), A t=4 \times 10^{-2}$ at $\beta=83^{\circ}(\boldsymbol{\vee}), A t=10^{-2}$ at $\beta=83^{\circ}(\mathbf{\square})$, all with $\nu=1\left(\mathrm{~mm}^{2} . \mathrm{s}^{-1}\right) \mathrm{s}$. The broken line shows $\hat{V}_{f} / \hat{V}_{t}=0.7+0.595 F r+0.362 F^{2}$. b) Normalized front velocity, $\hat{V}_{f} / \hat{V}_{\nu} \cos \beta$, as a function of normalized mean flow velocity, $\hat{V}_{0} / \hat{V}_{\nu} \cos \beta$. Data points with the same symbols belong to same experimental sequence: increasing Reynolds number through $\hat{V}_{0}$. The heavy solid line indicates the scaled front velocity from the lubrication model. The thin solid line shows $\hat{V}_{f}=\hat{V}_{0}$. The broken lines show our inertial exchange approximation through the simple model.

comparison of front velocity data in the exchange flow regime with the prediction:

$$
\frac{\hat{V}_{f}}{\hat{V}_{t}}=0.7+0.595 F r+0.362 F r^{2}
$$

The collapse of the data with respect to $F r$ is evident and the approximation is quite reasonable.

To explore the validity of the approximation (2.10) as $\chi$ decreases, we plot in Fig. 7b the same data but normalised with the viscous scale (e.g. as in Fig. 6). The broken curves now denote (2.10), which is different for different experimental sequences. However, the curves appear to converge in this figure close to the critical value $\hat{V}_{0} / \hat{V}_{\nu} \cos \beta=2 / \chi_{c}$ which is marked, and diverge thereafter. Note however, that in our experiments we have observed that even inertial exchange flows become viscous on increasing $\hat{V}_{0}$ (see Figs. 3 $\& 4$ earlier). Thus, above the critical $\hat{V}_{0} / \hat{V}_{\nu} \cos \beta=2 / \chi_{c}$ our experimental sequences are fitted well by (2.7).

\subsection{Overall classification of the flow regimes}

We are now in a position to summarise the observed flow regimes. In contrast to Taghavi et al. $(2010,2011)$ we are able to give quantitative predictions of both where each flow regime is found and the leading front velocity. We plot our experimental results in the $(F r, R e \cos \beta / F r)$-plane (equivalently the $\left(\hat{V}_{0} / \hat{V}_{t}, \hat{V}_{\nu} \cos \beta / \hat{V}_{t}\right)$-plane). Note that lines of constant $\chi$ correspond to linear rays through the origin, in the positive quadrant. Figure 8 plots the data from the full range of our experiments, as described in Table 1. Each experiment has been classified according to the classifications (s1-s3) of Taghavi et al. (2011). Only data satisfying $R e<2300$ has been used. The critical value $\chi_{c}=116.32 \ldots$ corresponds to the line

$$
\frac{\operatorname{Re} \cos \beta}{F r}=\frac{\chi_{c}}{2} F r,
$$




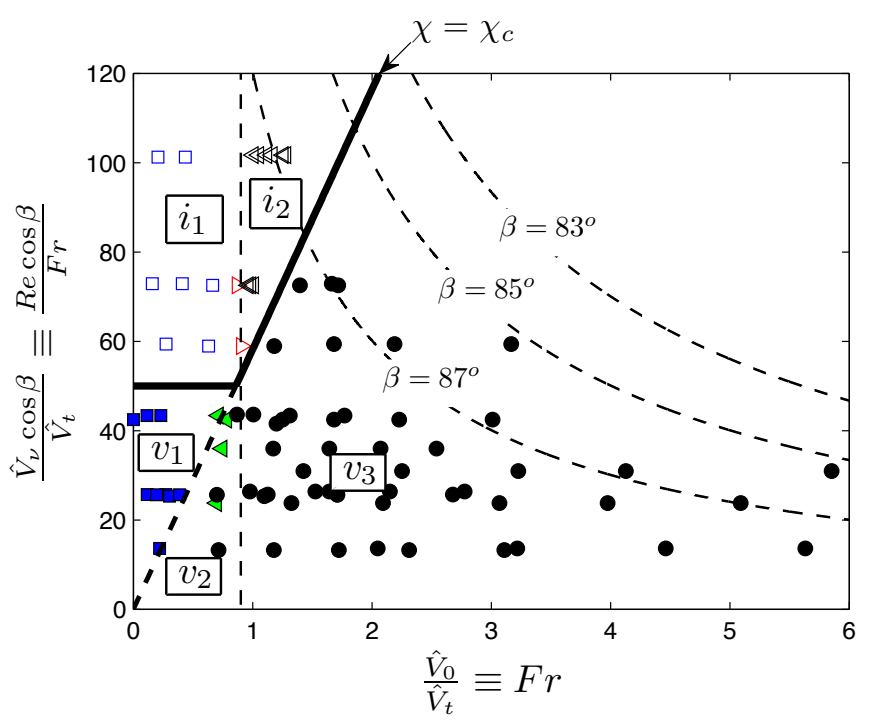

FiguRE 8. Classification of our results for the full range of experiments in the first and second regimes (p1 \& p2) from in Table 1, with secondary classifications: (s1) sustained back flow $(\mathbf{\square}, \square)$, (s2') stationary interface $(\triangleright),(\mathbf{s 2})$ temporary back flow $(\triangleleft, \triangleleft)$ and $(\mathbf{s 3})$ instantaneous displacement $(\bullet)$. Data point with filled symbols are viscous and with hollow symbols are inertial. The horizontal bold line shows the first order approximation to the inertial-viscous transition $\left(R e_{t} \cos \beta=50\right.$, from Seon et al. $\left.(2007 b)\right)$. The dotted line and its continuation (the heavy line) represent the prediction of the lubrication model for the stationary interface, $\chi=\chi_{c}$. The vertical dashed-line is $\hat{V}_{0} / \hat{V}_{t}=0.9$. The thin broken lines are only illustrative and show an estimate for the turbulent shear flow transition, implying to the third fully mixed regime. These are based on $R e=2300$. Regions marked with $v_{j}(\mathrm{j}=1,2,3)$ correspond to viscous regimes, and those marked $i_{j}(\mathrm{j}=1,2)$ are inertial regimes, as explained in the main text.

which is also marked in Fig. 8. The quantity $R e \cos \beta / F r=R e_{t} \cos \beta$, which is a Reynolds number based on the inertial velocity scale; see Seon et al. (2007b). This quantity is independent of $\hat{V}_{0}$. The dividing line $\left(R e_{t} \cos \beta=50\right)$ between viscous and inertial exchange flows is marked in Fig. 8 with the heavy solid horizontal line; see (2.8). Finally, at sufficiently large imposed velocities we expect to transition to the mixed p3 regime (e.g. see Fig. 3). A sufficient condition for this would be the onset of turbulence. Assuming that at high $R e$ the buoyancy effects have minimal effect, we might assume transition at a nominal value $R e=2300$. For different pipe inclinations these curves are marked in Fig. 8 with thin broken lines. At each angle the corresponding displacement data lies under the appropriate curve.

With reference to Fig. 8 we can identify the following regimes.

(a) Inertial exchange flow dominated regime: This regime is found for $R e_{t} \cos \beta \gtrsim$ 50 and for $F r=\hat{V}_{0} / \hat{V}_{t} \lesssim 0.9$. In Fig. 8 this regime is marked by $i_{1}$. This flow is characterized by development of Kelvin-Helmholtz-like instabilities and partial mixing. Buoyancy forces are sufficiently strong for there to be a sustained back flow. The front velocity scales with $V_{t}$ and is approximated reasonably well by (2.10).

(b) Inertial temporary back flow regime: In Fig. 8 this regime is marked by $i_{2}$ and is bounded by (2.11) and $F r=\hat{V}_{0} / \hat{V}_{t} \gtrsim 0.9$. On increasing the imposed flow $\hat{V}_{0}$, the destabilizing influences of inertia become progressively less efficient. The bulk flow remains generally inertial up until the critical stationary interface flow is encountered, 
along (2.11), after which the flow becomes progressively laminar. The front velocity scales with $V_{t}$ and is approximated reasonably well by (2.10).

(c) Viscous exchange flow dominated regime: Observed for $R e_{t} \cos \beta \lesssim 50$ and $\chi>\chi_{c}$. When the pure exchange flow is viscous it appears that the displacement flow obtained by adding a small imposed flow $\hat{V}_{0}$ is also viscous at long times. This regime is marked by $v_{1}$ in Fig. 8 . In this regime inertial effects can be observed at the beginning of displacement (i.e. at short time) where they limit the velocity of the trailing front moving upstream. Inertial effects are also significant local to the displacement front, where they usually appear in the form of an inertial bump. However in the bulk of the flow, energy is dissipated by viscosity. The front velocity can be well predicted by (2.7).

(d) Viscous temporary back flow regime: These flows are found in a regime bounded by (2.11) and $F r=\hat{V}_{0} / \hat{V}_{t} \lesssim 0.9$, marked by by $v_{2}$ in Fig. 8 . As with regime $i_{2}$ this regime is transitionary showing a progressive change from exchange-dominated to imposed flow-dominated as $F r$ is increased. The boundary of this regime with the exchange flow dominated regime occurs along (2.11), where stationary residual layers are found; see Taghavi et al. (2011). This is again a viscous regime and the front velocity can be well predicted by (2.7).

(e) Imposed flow dominated regime: When the imposed velocity is sufficiently strong, for either the inertial or viscous exchange flow dominated regimes, the flow transitions to a laminarised state dominated by viscous effects. For the inertial exchange flow, the stabilizing effect is seen on the whole flow while in the viscous exchange flow, the stabilizing effect is observed through the spreading out of the inertial bump at the front. The front velocities in this regime are predicted by (2.7). In Fig. 8 this regime is marked by $v_{3}$.

( $f$ ) Mixed/turbulent regime: We have not studied in detail this final transition, but for $A t \ll 1$ we expect that the transitional Reynolds numbers should be approximately the same as for the transitional flow of a single fluid in pipe (as we are iso-viscous and miscible). In this regime, the front velocity is approximately equal to the imposed flow velocity for our experiments, but at longer times we would expect dispersion to have significant effects.

To graphically illustrate the above predictions as $\hat{V}_{0}$ is increased from zero, we present two experimental sequences in Fig. 9. In Fig. 9a we are in the viscous regime initially. The lubrication approximation is very good for $\chi>\chi_{c}$ and only begins to diverge as the stationary interface regime is passed for increasing $\hat{V}_{0}$. Looking at the inset pictures below the critical stationary interface, it is clear that the imposed flow changes the shape of the inertial bump at the displacement front. After the critical stationary interface is attained, the inset figures show that the inertial bump is absent. The divergence of $\hat{V}_{f}$ from the lubrication prediction as $\hat{V}_{0}$ increases suggests that inertial effects in the flow are becoming important (see $\S 4$ ). Figure $9 \mathrm{~b}$ shows data from a sequence in which we are initially in the inertial exchange flow regime. The solid line now shows the approximation with (2.10), which is again very good $\chi>\chi_{c}$. For $\chi \leqslant \chi_{c}$ we observe that the imposed flow gradually laminarises the flow. The model (2.10) is no longer a good approximation and the viscous lubrication approximation takes over.

\section{Displacement in channels}

As a second displacement flow geometry we consider a plane channel. Whereas in the pipe flow any detailed computations would necessarily be three-dimensional, in the plane channel they are two-dimensional, which has distinct advantages in terms of computational speed. Furthermore the simpler geometry allows room for analysis that would be 

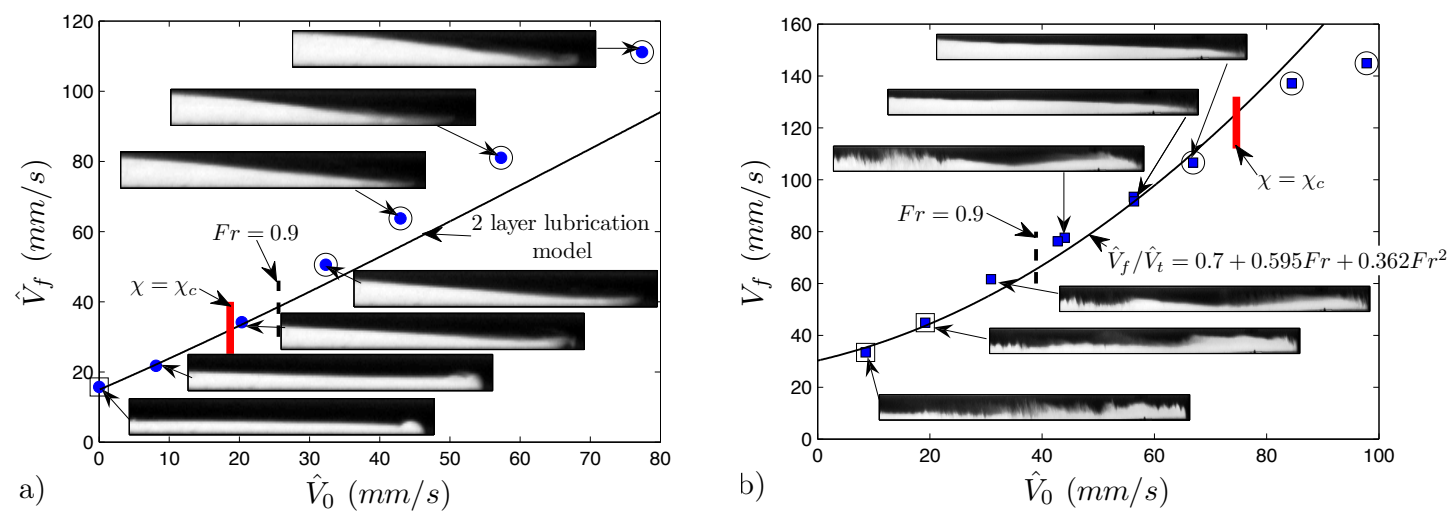

FigURE 9. Example comparison of the engineering predictions of the front velocity $\hat{V}_{f}$ with experimental data: a) viscous regime for the pure exchange flow; $\beta=85^{\circ}, A t=3.5 \times 10^{-3}$, $\left.\nu=1\left(\mathrm{~mm}^{2} \cdot \mathrm{s}^{-1}\right),\left(R e_{t} \cos \beta \approx 42\right) ; \mathrm{b}\right)$ inertial regime for the pure exchange flow; $\beta=83^{\circ}$, $A t=10^{-2}, \nu=1\left(\mathrm{~mm}^{2} \cdot \mathrm{s}^{-1}\right)$. Sustained back flows and instantaneous displacements are marked by the superposed squares and circles respectively. Data points without marks are either temporary back flows, stationary interfaces or undetermined experiments (i.e. insufficient experiment time or short pipe length above the gate valve). The thin line shows the prediction of lubrication model in a) and of (2.10) in b). The thick vertical line shows the prediction of stationary interface from the lubrication model. The thick vertical broken line shows the prediction of the transition between temporary back flow and instantaneous displacement, through $\hat{V}_{0} / \hat{V}_{t}=0.9$. The insets are pictures of a section of tube a few centimeters below the gate valve in the corresponding flow domains: (264 ( $\mathrm{mm})$ long in a), 1325 ( $\mathrm{mm})$ long in b)).

prohibitively complex in the pipe geometry; see $\S 4$ later. One can either consider the plane channel as an independent study or as one which allows new perspectives on the pipe displacement flow.

In making inferences regarding the pipe flow some caution is needed. For example Hallez \& Magnaudet (2008) studied pure exchange flows in pipes and channels in the inertially dominated regime, when the fluids mix, and have shown distinct differences in the flow structures observed. Therefore, direct comparisons are only like to be valid in regimes where viscous forces dominate in balancing buoyant and imposed pressure drops.

\subsection{Computational methodology}

In place of physical experiments, we have carried out a number of numerical simulations of $2 \mathrm{D}$ displacements in an inclined plane channel. The geometry and notation are as represented in Fig. 1. The computations are fully inertial, solving the full 2D NavierStokes equations with phase change modelled via a scalar concentration, $c$. The system is given by (1.2)-(1.4). The equations (1.2)-(1.4) have been discretised using a mixed finite element/finite volume method. The numerical method is exactly as described in Taghavi et al. (2011). Approximately 400 simulations have been carried out, as detailed in Table 2. We have selected a range of dimensional parameters that is similar in scope to those of our pipe flow experiments. However, we have not explored very high $R e$ (typically $R e<500$ for our simulations). At larger $R e$ we would expect to enter a fully mixed turbulent regime, for which we have not explored the performance of our code.

After running each simulation, the front velocities were calculated from the spatiotemporal plot of $c$, i.e. mimicking the experimental procedure. Mesh refinement was carried out until successively calculated front velocities on meshes differed by $1-4 \%$, (over the range of physical parameters explored). The meshes used for the computations presented 


\begin{tabular}{|c|c|c|c|c|c|}
\hline$\beta^{\circ}$ & $\hat{\nu}\left(\mathrm{mm}^{2} \cdot \mathrm{s}^{-1}\right)$ & At $\left(\times 10^{-3}\right)$ & $\hat{V}_{0}\left(\mathrm{~mm} \cdot \mathrm{s}^{-1}\right) \|$ & $R e$ & $F r$ \\
\hline 81 & 1 & 3.5 & $0-27$ & $0-500$ & $0-1.03$ \\
\hline $83 \dagger$ & $1-2$ & $1-10$ & $0-153$ & $0-2907$ & $0-3.53$ \\
\hline 85 & $1-2$ & $1-10$ & $0-27$ & $0-500$ & $0-1.92$ \\
\hline 87 & $1-2$ & $1-10$ & $0-27$ & $0-500$ & $0-1.92$ \\
\hline 88 & 1 & 3.5 & $0-27$ & $0-500$ & $0-1.03$ \\
\hline 89 & 1 & 10 & $0-27$ & $0-500$ & $0-0.61$ \\
\hline 90 & $1-2$ & $0-10$ & $0-27$ & $0-500$ & $0-\infty$ \\
\hline & \multicolumn{5}{|c|}{$\begin{array}{c}\dagger \text { Most of the simulations were conducted in the range of } \\
{\left[\hat{\nu}\left(\mathrm{mm}^{2} \cdot \mathrm{s}^{-1}\right), A t\left(\times 10^{-3}\right), \hat{V}_{0}\left(\mathrm{~mm}_{\mathrm{s}}{ }^{-1}\right) ; R e, F r\right] \in} \\
{[1-2,1-10,0-27 ; 0-500,0-1.92]}\end{array}$} \\
\hline
\end{tabular}

below have 28 cells across the channel, refined slightly towards the walls, and 400 cells along the length of the channel. We acknowledge that the meshes used are relatively coarse, but note that the principle information being extracted from the simulations is bulk information, e.g. spatiotemporal plots and front speeds. These features are less sensitive to refinement, which would be advisable if e.g. flow instabilities and mixing were to be directly studied.

\subsubsection{Code benchmarking}

Various simple test problems have been implemented. The code has also been benchmarked against representative numerical and experimental studies. For example, we have found good agreement in computed front velocities with the results of Sahu et al. (2009b); (also in private communications with Matar \& Sahu). We also observe similar qualitative behavior in the displacement flow behind the front. The onset of small interfacial waves appears to occur in our simulations at slightly higher values of the imposed flows than with their code.

We have also compared our results with those of Hallez \& Magnaudet (2008) for exchange flow in a $2 \mathrm{D}$ channel over the range $\beta=60-90^{\circ}$, and capture all the main trends and qualitative behaviors. The emphasis in Hallez \& Magnaudet (2008) is on the initial slumping phase (which is also inertial) and on quantifying the details of mixing and instability. They have consequently considered shorter channel lengths $(32 \times \hat{D})$ and shorter computational times than we have. For example, we observe the strong influence of vortices periodically cutting the channels of pure fluid which feed the advancing fronts and help to maintain constant front velocity. In near-horizontal channels we have observed an initial inertial phase during which the front velocity rapidly increases to an approximately constant plateau. Afterwards, viscous effects come into play and the front velocity decreases and attains a final velocity. On increasing the angle from horizontal, we observed a slight increase in the front velocity and found a constant plateau of modified Froude number versus tilt angle between $\beta=70-80^{\circ}$; see e.g. Fig 5 in Hallez \& Magnaudet (2008). However, some quantitative differences exist. For example, our front velocities were $10-15 \%$ lower than values reported by Hallez \& Magnaudet (2008), who anyway commented that their front velocities were larger than expected (see figures 4 or 7 in their work), by comparison e.g. with the corresponding experiments performed by Seon et al. $(2004,2005,2006,2007 b)$. This difference is at least partly attributed to the short timescale of the numerical experiments in Hallez \& Magnaudet (2008). 


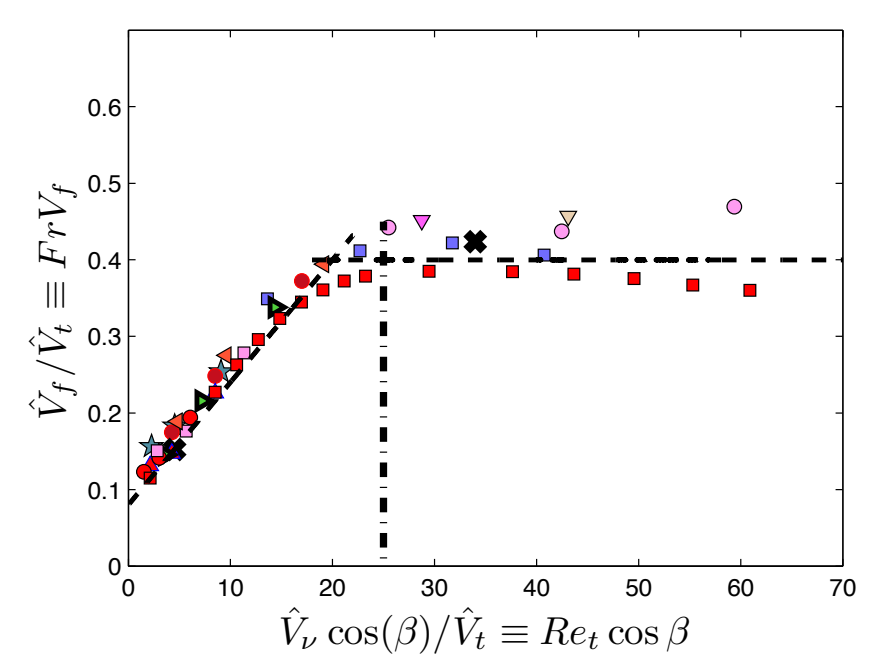

Figure 10. Variation of the normalised stationary front velocity $\hat{V}_{f} / \hat{V}_{t}$ as a function of the inertial Reynolds number $R e_{t} \cos \beta=\hat{V}_{\nu} \cos \beta / \hat{V}_{t}$ for lock-exchange flows $\left(V_{0}=0\right)$. The simulation data correspond to different tilt angles, viscosities and density contrasts in the range of $\left[\beta^{\circ}, \hat{\nu}\left(\mathrm{mm}^{2} \cdot \mathrm{s}^{-1}\right), A t\left(\times 10^{-3}\right)\right] \in[60-89,1-4,1-10]$. The transition between viscous and inertial lock-exchange flows for the mentioned simulation range occurs at $R e_{t} \cos \beta=25 \pm 5$. Guide lines are drawn in this figure: horizontal dashed line at $\hat{V}_{f} / \hat{V}_{t}=0.4$, vertical dash-dot line at $R e_{t} \cos \beta=25$, and the oblique dashed line showing a more or less linear relation between $\hat{V}_{f}$ and $\hat{V}_{\nu} \cos \beta$.

Apart from these comparisons, the same code has been used extensively in Hormozi et al. (2011) where it has been benchmarked against the recent experiments of d'Olce (2008); d'Olce et al. (2008), in which miscible core-annular Newtonian flows of differing viscosities develop pearl and mushroom shaped instabilities. Good quantitative comparisons were made.

\subsection{Exchange flow results}

We first start with an examination of the pure exchange flow in the $2 \mathrm{D}$ channel. The objective is to gain an understanding of the transition from inertial to viscous dominated exchange flows $\left(\hat{V}_{0}=0\right)$, parallel to that deduced in the experimental studies of Seon et al. $(2007 b)$. We have seen the relevance of this transition for pipe flows as a first order prediction of the transition from viscous to inertial flows in buoyancy dominated displacement flows $\left(\hat{V}_{0}>0\right)$. The exchange flow results have also given the leading order term in the expansion (2.10). Our results are shown in Fig. 10 where we have plotted the normalized front velocity $\hat{V}_{f} / \hat{V}_{t}$ against the inertial Reynolds number $R e_{t} \cos \beta=$ $\hat{V}_{\nu} \cos \beta / \hat{V}_{t}$.

The transition between viscous and inertial lock-exchange flows in plane channels, as determined by our $2 \mathrm{D}$ simulations, occurs in the range $R e_{t} \cos \beta=25 \pm 5$. We see a separation between a linear increase of $\hat{V}_{f}$ with the viscous velocity scale $\hat{V}_{\nu} \cos \beta$, for $R e_{t} \cos \beta<25 \pm 5$ (viscous regime) and a constant plateau for which $\hat{V}_{f} \approx 0.4 \hat{V}_{t}$ for the range of flow parameters studied. This compares with values of $R e_{t} \cos \beta \approx 50$ and $\hat{V}_{f} \approx 0.7 \hat{V}_{t}$ for the pipe exchange flow transition; see Seon et al. (2006). As $R e_{t} \cos \beta$ increases, the decrease in $\hat{V}_{f}$ is due to (geometry dependent) coherent vortices which cut the channel of pure fluid feeding the front. this finally decreases the density contrast at 



FigURE 11. Variation of the downstream front velocity $\hat{V}_{f}$ as a function of mean flow velocity $\hat{V}_{0}$ for different inclination angles: a) $\beta=83^{\circ}$; b) $\beta=87^{\circ}$; In each plot: $A t=10^{-2}$ $(\boldsymbol{\square}), A t=3.5 \times 10^{-3}(\bullet), A t=10^{-3}(\mathbf{\Delta})$, all with $\nu=1\left(\mathrm{~mm}^{2} \cdot \mathrm{s}^{-1}\right) ; A t=3.5 \times 10^{-3}$ with $\nu=2\left(\mathrm{~mm}^{2} \cdot \mathrm{s}^{-1}\right)(\boldsymbol{\nabla})$. In all plots sustained back flows and instantaneous displacements are squared and circled respectively. The heavy solid line is $\hat{V}_{f}=1.5 \hat{V}_{0}$.

the front and therefore the front velocity. This interesting phenomenon has been studied in depth by Hallez \& Magnaudet (2008). The extent of the plateau (in $R e_{t} \cos \beta$ ) is not however known. To investigate this regime would require a detailed study of mixing regimes occurring at inclinations closer to vertical. This is not the present objective.

\subsection{Displacement flow results}

Turning now to the displacement flow results, an overall comment is that there are many aspects of the flow that are qualitatively similar to the pipe flow, but also significant differences. Starting with bulk flow parameters, such as the leading displacement front, there is qualitatively similar behaviour as $\hat{V}_{0}$ is increased from zero. Figure 11 shows the variation of $\hat{V}_{f}$ with $\hat{V}_{0}$ for a two of the inclination angles studied, at different Atwood numbers $A t$ and kinematic viscosities $\hat{\nu}$. Low values of $\hat{V}_{0}$ are dominated by exchange flow characteristics (p1) and an approximate plateau in $\hat{V}_{f}$ is observed. On increasing $\hat{V}_{0}$ we enter a regime, where the increase in $\hat{V}_{f}$ is approximately linear with $\hat{V}_{0}$, i.e. (p2). These two regimes occur at different inclinations, very similar to the pipe flows. As well as the transition from exchange flow dominated to linear regime, consideration of the trailing front leads to a secondary classification $(\mathbf{s} 1 \mathbf{- s 3})$ that ranges from sustained back flow through instantaneous displacement, exactly as for the pipe flow, as explored in detail in Taghavi et al. (2011). Other features of the flow where we find similarity with the pipe flow are as follows.

- Inertial effects are more prevalent as $A t$ increases and at steeper channel inclinations.

- Imposition of the mean flow does have a laminarising effect on the flow. For example, we can observe an inertial region at the leading front that is strongly affected by the imposed flow. However, we have not observed strongly inertial exchange flows being fully stabilized, which was the case in the pipe flows.

- In the exchange flow dominated regime, whether inertial or viscous, the basic structure is a two-layer flow bounded by one front moving downstream and a second front moving upstream.

We now discuss some of the significant differences with the pipe displacement flows, starting with viscous flows (defined approximately by $R e_{t} \cos \beta<25 \pm 5$ ) and considering the imposed flow dominated regime. The most obvious difference is that the front is 

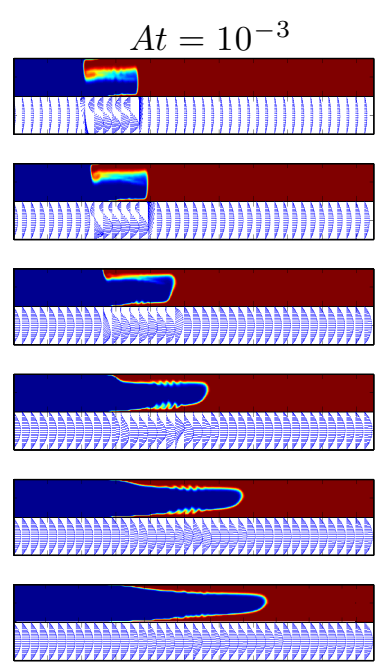

At $=3.5 \times 10^{-3}$

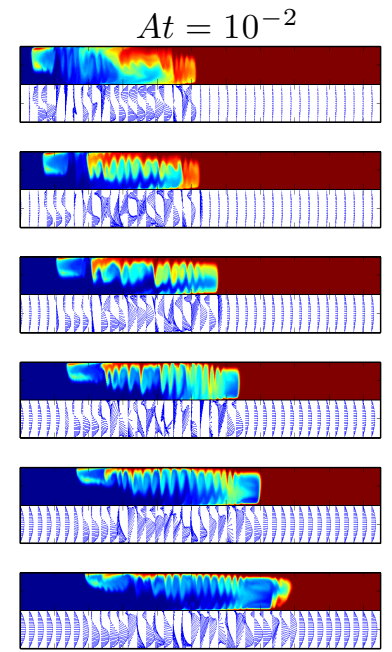

FiguRE 12. Panorama of concentration colourmaps and velocity vectorfields for displacements with $\nu=1\left(\mathrm{~mm}^{2} \cdot \mathrm{s}^{-1}\right)$, each taken at $\hat{t}=25(\mathrm{~s})$ for inclination $\beta=83^{\circ}$. In each panel the rows from top to bottom show $\hat{V}_{0}=2.7,5.3,10.5,15.8,21.0,26.3\left(\mathrm{~mm} . \mathrm{s}^{-1}\right)$ (equivalently $R e=50,100,200,300,400,500$ ). The columns from left to right show $A t=10^{-3}, 3.5 \times 10^{-3}, 10^{-2}$. The length shown is the whole channel, $\hat{L}=100 \hat{D}$.

not observed to displace in a slumping 2-layer pattern, but instead a finger advances approximately along the centre of the channel leaving behind upper and lower layers of displaced fluid. Typical examples are shown in Fig. 12 where we show snapshots of the concentration profile and velocity vectorfields at $\hat{t}=25$ (s) for different $A t$ and imposed $\hat{V}_{0}$. This feature is partly expected. In parallel with our experimental study, we have considered low viscosity fluids (essentially water) and hence enter the viscous regime by ensuring that $A t$ is small and $\beta$ is close to horizontal. Obviously, on taking $A t \rightarrow 0$ we have two identical fluids and expect to recover a plane Poiseuille flow. This is indeed the case. The smallest values of Atwood number $\left(A t=10^{-3}\right)$ correspond to a $0.2 \%$ density difference between fluids and it is hardly surprising to see the front nearly symmetric and advancing close to the channel centreline. For two identical fluids in plane Poiseuille flow the leading front speed would be simply $\hat{V}_{f}=1.5 \hat{V}_{0}$, which is the heavy solid line marked in Fig. 11, (note this is $\hat{V}_{f}=4 / 3 \hat{V}_{0}$ for the pipe, if a stratified interface is assumed). We can see that as the linear regime is entered the front velocity lies just below this iso-dense limit.

Although the front advances towards the centre of the channel, density differences are expressed through asymmetry of the residual layers above and below. Typically the lower layer is shorter and thinner than the upper layer, except near the tip where it seems that inertial effects act to point the tip upwards. This is the analogy of the inertial bump that we have observed in the pipe displacement flows. In the context of the plane channel results, it is interesting to review the pipe flow displacement experiments again. For very similar physical parameters we always observed a slumping displacement front. A possible explanation for this would be that the pipe allows for three-dimensional secondary flows, i.e. less dense fluid in a layer underneath an advancing finger can be squeezed azimuthally around the sides of the tube by the heavier finger. In a strictly 2D geometry this does not 

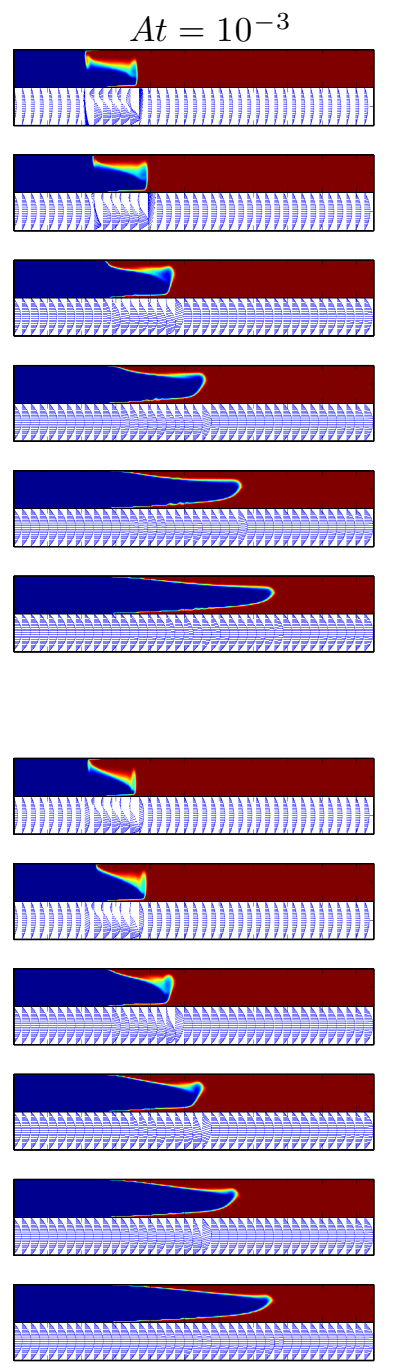
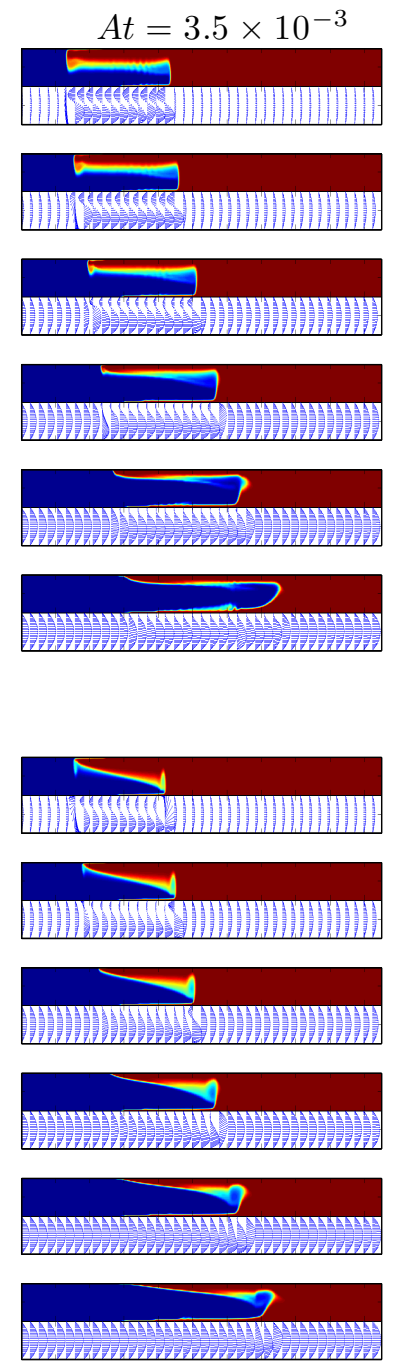

$A t=10^{-2}$


Figure 13. As Fig. 12, except for $\beta=87^{\circ}$ (top) and $\beta=90^{\circ}$ (bottom).

happen. In this context it would be interesting to study displacements in a rectangular cross-sectional channel.

To give a broader understanding of the flow variations with $A t, R e$ and $\beta$, Fig. 13 presents a panorama of concentration colourmaps and velocity vectorfields for two further pipe inclinations, to compare with Fig. 12. We can see a clear distinction between flows that are predominantly viscous and those that are inertial. For the viscous regime the interface is well defined, although we do see dispersive mixing from secondary flows associated with the inertial tip, close to each front. Within this class of flows, increased At and more horizontal channels tend to push the finger towards the lower wall of the channel. We should note that the lower interface of the finger is density unstable and for some simulations we can observe small instabilities developing, possibly of RayleighTaylor type. The velocity profiles in Figs. $12 \& 13$ show how 2D effects are progressively 

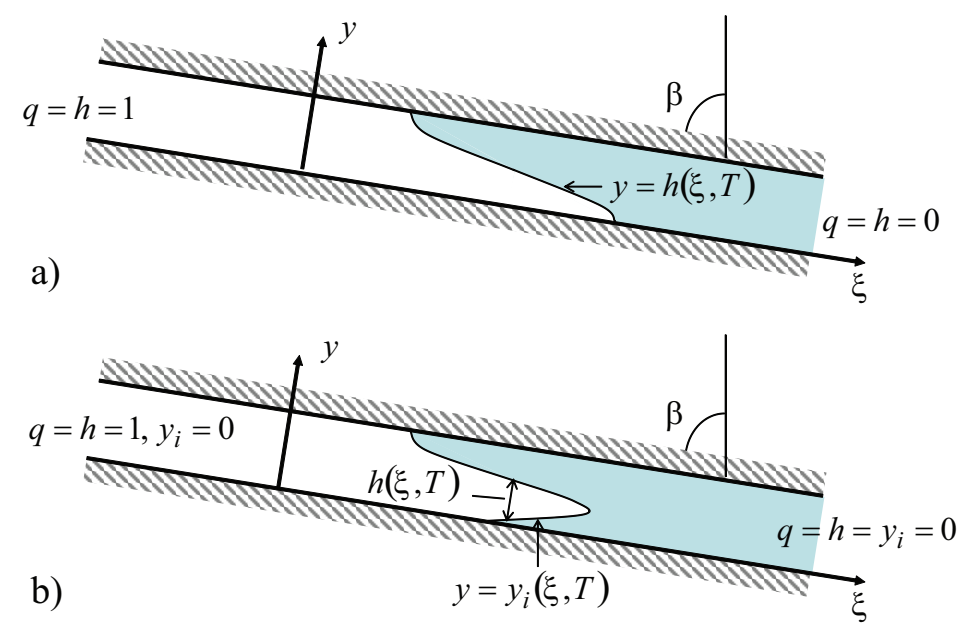

FiguRE 14. Schematic of the displacement geometry: a) 2-layer model; b) 3-layer model.

important at increasing $A t$ and in steeper channels. The unsteadiness of the velocity field is clearly confined to the mixed region where both fluids are found. Outside of this region the laminar Poiseuille flow is quickly re-established.

At fixed inclination, increasing $A t$ leads to the inertial regime, where we observe KelvinHelmholtz like instabilities along the top (and to a lesser extent bottom) interface; see e.g. $\beta=83^{\circ}, A t=10^{-2}$ in Fig. 12 . The degree of mixing is reduced as $\hat{V}_{0}$ increases, but the flows remain obviously inertial. For the larger $A t$ the thin lower residual layer observed in the viscous regime is essentially washed away. Referring to Fig. 11 (which includes data from the inertial flows in Figs. 12 \& 13) it is noteworthy that even when the imposed flow dominated regime is inertial, the increase in front velocity with $\hat{V}_{0}$ still becomes approximately linear as $\hat{V}_{0}$ increases. Note that the entire length of channel (length $\hat{L}=100 \hat{D}$ ) is shown in Figs. $12 \& 13$, so some caution needs to be exercised in interpreting apparently high frequency flow features; these instabilities are in fact of order unity wavelength. An increase in $\nu$ serves mainly to stabilise the displacement, promoting the viscous regime as might be expected. Similar stabilisation also comes from making the channel progressively more horizontal.

\subsection{Quantitative prediction of the front velocity}

As with the pipe flows, we would like to be able to approximate the front velocities using relatively simple models describing the longer time evolution of the front. We follow a similar strategy as with the pipe displacement flows.

\subsubsection{Lubrication/thin film style models}

In Taghavi et al. (2011) we have focused at the transition between exchange dominated flows and imposed flow dominated flows. Data from channel flow simulations was analysed with the aid of the lubrication model of Taghavi et al. (2009). Only simulations from the viscous regime were analysed and the lubrication model prediction of the transition to imposed flow from exchange flow was found to be very accurate. The lubrication model in Taghavi et al. (2009) is based on the assumption that the heavier fluid will slump towards the bottom of the channel and displace the fluid in this slumping configuration. This leads to an evolution model for the interface height, analogous to that considered 
earlier for the pipe, i.e.

$$
\frac{\partial h}{\partial T}+\frac{\partial}{\partial \xi} q\left(h, h_{\xi}\right)=0
$$

(see Fig. 14a). The flux function $q\left(h, h_{\xi}\right)$ depends again only on the single dimensionless parameter $\chi$. The long-time hyperbolic limit of this model has a single critical value $\chi=\chi_{c}=69.94$ at which the upper layer of displaced fluid has a stationary interface. The efficacity of (3.1) in describing this transition is undoubtedly due to the fact that for viscous exchange flows the $2 \mathrm{D}$ computations evolve into two-layer type slumping flows. However as we have seen, imposed flow dominated displacements generally propagate along the channel centre, being pushed towards the lower wall by increasing $A t$ and $\beta$. Although the flow is long and thin, the two-layer topology of Taghavi et al. (2009) is not what is observed.

Instead a three-layer structure is more appropriate, see Fig. 14b, and after assuming such a structure it is fairly straightforward to derive a lubrication style model for the interface evolution. Now we have two interfaces, as illustrated, and (3.1) is replaced by:

$$
\begin{aligned}
\frac{\partial h}{\partial T}+\frac{\partial}{\partial \xi} q\left(h, y_{i}, h_{\xi}, y_{i, \xi}\right) & =0, \\
\frac{\partial y_{i}}{\partial T}+\frac{\partial}{\partial \xi} q_{L}\left(h, y_{i}, h_{\xi}, y_{i, \xi}\right) & =0 .
\end{aligned}
$$

The height $y_{i}(\xi, T)$ is the height of the lower interface and $h(\xi, T)$ reflects the thickness of the displacing central finger, as illustrated. The two flux functions are defined as:

$$
q=\int_{y_{i}}^{y_{i}+h} u \mathrm{~d} y, \quad q_{L}=\int_{0}^{y_{i}} u \mathrm{~d} y .
$$

For Newtonian fluids it is straightforward to calculate these as functions of the interface heights and of the parameter $\chi$, (see appendix A). Note that these functions are only defined for $y_{i} \in[0,1]$ and $h \in\left[0,1-y_{i}\right]$, since $y_{i}+h$ denotes the height of the upper interface. As $y_{i} \rightarrow 0$ the two-layer model is recovered.

If again we consider the long time dynamics of a centrally propagating finger, the system (3.2) \& (3.3) is made more simplified neglecting the spreading effects of $h_{\xi}$ and $y_{i, \xi}$ and considering the remaining hyperbolic system. Necessary conditions to have a steadily propagating wave are that:

$$
h \frac{\partial q}{\partial h}\left(h, y_{i}, 0,0\right)=q\left(h, y_{i}, 0,0\right), \text { and } \frac{\partial q}{\partial h}\left(h, y_{i}, 0,0\right)=\frac{\partial q_{L}}{\partial y_{i}}\left(h, y_{i}, 0,0\right) .
$$

The flux functions can be analysed numerically to compute values of $\left(h, y_{i}\right)$ for which we have solutions to the above 2 conditions, at each value of $\chi$. Sometimes there are multiple solutions to (3.5), but it is relatively simple to discount some of the solutions on physical grounds.

Having solved (3.5) we have a prediction to the front velocity, say $V_{3, f}(\chi)$, that comes from the 3-layer viscous lubrication model (3.2) \& (3.3), and which depends only on $\chi$. Similarly, analysis of (3.1) gives a front velocity prediction, say $V_{2, f}(\chi)$, from the two layer model of Taghavi et al. (2009), that also depends only on $\chi$. These predictions are compared with the normalised front velocities $\hat{V}_{f} / \hat{V}_{\nu} \cos \beta$, from our 2D numerical computations in Fig. 15. The transition between exchange flow dominated flows and imposed flow dominated flows is marked by the circle in Fig. 15 and is at $\chi_{c}=69.94$. To the right of this point, in the range $\chi \simeq 23-24$, the two front velocity predictions intersect: $V_{3, f}(\chi)=V_{2, f}(\chi)$ and for smaller values of $\chi$ the 3-layer front velocity is larger. 


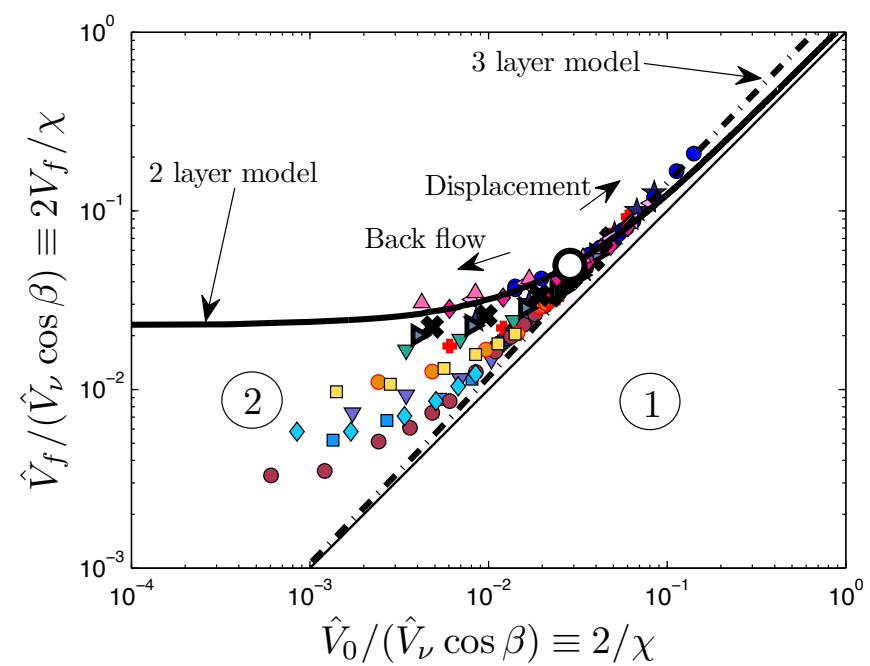

FiguRE 15. Normalized front velocity, $\hat{V}_{f} / \hat{V}_{\nu} \cos \beta$, as a function of normalized mean flow velocity, $\hat{V}_{0} / \hat{V}_{\nu} \cos \beta$, for the full range of simulations in regimes $\mathbf{p} 1 \& \mathbf{p} 2$, (i.e. $R e<500$ in Table 2). Data points with the same symbols belong to computational sets of increasing mean velocity $\hat{V}_{0}$ for fixed values of density contrast and/or viscosity. The heavy solid line indicates the scaled front velocity obtained by the 2-layer lubrication model (3.1). The circle on the heavy solid line shows the theoretical balance at which the stationary interface is found. The light solid line indicates the lower bound, $\hat{V}_{f}=\hat{V}_{0}$, below which front velocities are not possible (region denoted with the circled 1). The region denoted with the circled 2 represents flows with increasingly significant bouyancy effects. The heavy broken line represents the prediction of the scaled front velocity from the 3-layer lubrication model. The 2-layer and 3-layer model predictions intersect at $\chi \simeq 23-24$.

For viscous displacements, in the range of small $\chi$, where the imposed flow is dominant, the three-layer model does generally give a better prediction of front velocity than the 2-layer model. Nevertheless, the interface speed remains faster in the $2 \mathrm{D}$ computations than predicted by either of the lubrication models. This suggests that other effects such as inertia may also be significant in this range. It is also worth mentioning that for many of the imposed flow dominated cases computed numerically the $2 \mathrm{D}$ results are not cleanly represented by either lubrication model. As the flows evolve, secondary flows around the inertial tip of the leading front act to dispersively mix fluid over a significant region, so that the interface is not clearly defined. Secondly, we have seen that the lower interface is density unstable and can become vulnerable to density driven instabilities. At larger At or $\beta$, the lower layer thickness diminishes to the size of the mesh cell, or vanishes. In some respects, for many of the flows we are somewhere between a 2-layer and 3-layer model, e.g with a diffuse lower layer.

For larger values of $\chi>\chi_{c}$ as the exchange component becomes progressively stronger, the two-layer lubrication model gives a reasonable approximation to the front velocity for the viscous exchange flows, up to the transition at the stationary layer flow; see e.g. Taghavi et al. (2011). However, in Fig. 15 we also observe many data points for $\chi>\chi_{c}$ that fall below the 2-layer lubrication velocity prediction. In general, these points correspond to inertial exchange-dominated flows, which we now discuss. 



FiguRE 16. a) Normalized front velocity, $\hat{V}_{f} / \hat{V}_{t}$, as a function of normalized mean flow velocity, $\hat{V}_{0} / \hat{V}_{t}=F r$, plotted for simulation sequences in the pure inertial regime $30<R e_{t} \cos \beta<80$. Data points with the same symbols belong to same experimental sequence: increasing Reynolds number through $\hat{V}_{0}$. The broken line shows (3.6) and the dotted line shows (3.7), for $\mathrm{Fr}>1$. b) Normalized front velocity, $\hat{V}_{f} / \hat{V}_{\nu} \cos \beta$, as a function of normalized mean flow velocity, $\hat{V}_{0} / \hat{V}_{\nu} \cos \beta$ for different sets of simulations: $\beta=85^{\circ}, A t=10^{-2}, \nu=1\left(\mathrm{~mm}^{2} \cdot \mathrm{s}^{-1}\right)(\diamond) ; \beta=87^{\circ}$, $A t=10^{-2}, \nu=1\left(\mathrm{~mm}^{2} \cdot \mathrm{s}^{-1}\right)(\square) ; \beta=83^{\circ}, A t=3.5 \times 10^{-3}, \nu=2\left(\mathrm{~mm}^{2} \cdot \mathrm{s}^{-1}\right)(\boldsymbol{\nabla})$. The heavy solid line indicates the scaled front velocity from the lubrication model. The thin solid line shows $\hat{V}_{f}=\hat{V}_{0}$. The broken lines show the inertial exchange flow approximation (3.6).

\subsubsection{Inertia dominated flows}

In the range $R e_{t} \cos \beta>25 \pm 5$ (where exchange flows have been observed to be inertial), for positive $\hat{V}_{0}$ we also find inertial displacement flows. As with the pipe flows, the relevant velocity scale at zero imposed flow is the inertial velocity $\hat{V}_{t}$. As $\hat{V}_{0}$ is increased from zero the competition between $\hat{V}_{0}$ and $\hat{V}_{t}$ is captured in the Froude number $F r$, again suggesting that $\hat{V}_{f} / \hat{V}_{t}=f(F r)$. Taking a Taylor expansion of $f(F r)$, for small $F r$, in the style of (2.9), and comparing with the data for inertial exchange-dominated flows leads to the model:

$$
\frac{\hat{V}_{f}}{\hat{V}_{t}}=0.4+0.407 F r+0.704 F r^{2} .
$$

The initial coefficient is fitted from the pure exchange flow data only $(F r=0)$. The next two coefficients lie in the intervals $(0.347,0.467)$ and $(0.629,0.779)$, respectively, at confidence level of $95 \%$.

The expression (3.6) is derived based on small $F r$, in the exchange flow dominated regime. As Fr increases the imposed flow effects become dominant, but the flows remain inertial. As we have commented earlier, we recover a linear relationship between $\hat{V}_{f}$ and $\hat{V}_{0}$. This is approximated reasonably by the expression:

$$
\hat{V}_{f} \approx 1.5 \hat{V}_{0} .
$$

The transition between (3.6) and (3.7) takes place at $F r \approx 1$. Figure 16a plots our inertial regime data, normalised with $\hat{V}_{t}$. The similarity scaling is evident, i.e. the collapse of the data with respect to $F r$, and the approximation of (3.6) and (3.7) is very reasonable. Figure 16b plots the data in the variables of Fig. 15, showing that (3.6) effectively describes the inertial data below the 2-layer lubrication model. 


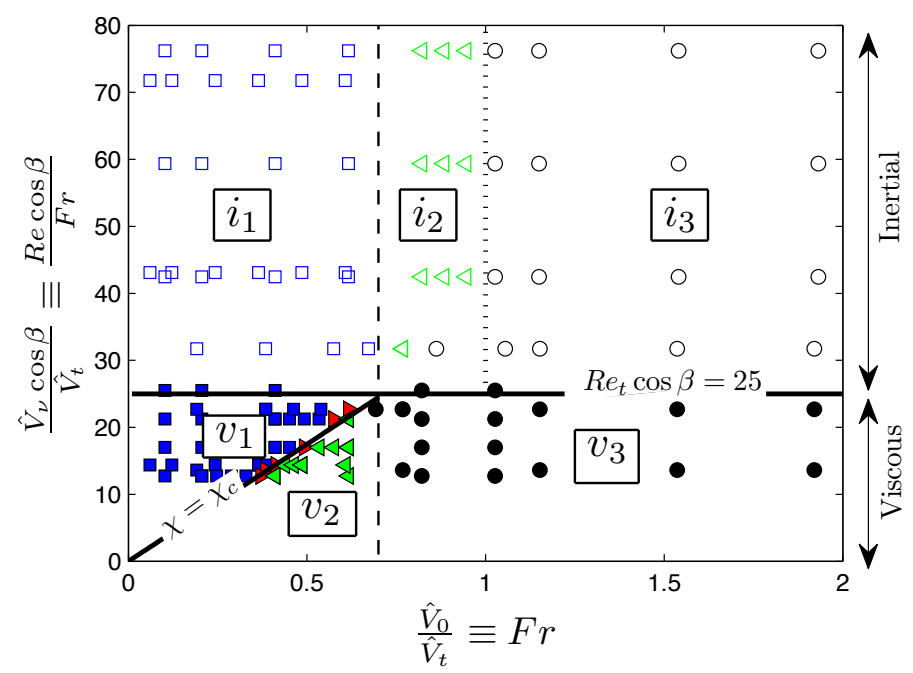

FiguRE 17. Classification of our results for the full range of simulations in the first and second regimes with laminar imposed flows: sustained back flow $(\mathbf{\square}, \square)$, stationary interface $(\bullet)$, temporary back flow $(\triangleleft, \triangleleft)$ and instantaneous displacement $(\bullet, \circ)$. Data point with filled symbols are viscous and with hollow symbols are inertial. The horizontal bold line shows the first order approximation to the inertial-viscous transition $\left(R e_{t} \cos \beta=25\right.$, from Fig. 10). The angled heavy line represents the prediction of the stationary interface from the two-layer lubrication model: $\chi=\chi_{c}$. The vertical dashed-line is $\hat{V}_{0} / \hat{V}_{t}=0.7$ and the dotted-line is $\hat{V}_{0} / \hat{V}_{t}=1$. Regions marked with $v_{j}$ and $i_{j}(\mathrm{j}=1,2,3)$ are viscous and inertial, respectively, as explained in the main text.

\subsection{Overall flow classifications and front velocity predictions}

Figure 17 presents data from the full range of simulations in regimes p1 \& p2, (note that our code is not suitable for high $R e$ shear flow transitions, i.e. p3). The secondary classifications s1-s3 are marked and we have also identified flows as either viscous or inertial. The most significant difference with the pipe flows (see Fig. 8) is that the criterion $\left(R e_{t} \cos \beta=25 \pm 5\right)$ separating inertial and viscous dominated flows appears to remain valid for $\hat{V}_{0}>0$. This criterion is derived from the pure exchange flow $\left(\hat{V}_{0}=0\right)$ and has been identified by finding where the viscous similarity scaling breaks down; see Fig. 10 . We classify the flows as follows:

(a) Viscous exchange flow dominated regime: this regime is defined approximately as flows satisfying $\chi>\chi_{c}$ and $R e_{t} \cos \beta<25 \pm 5$. In Fig. 17 this regime is marked by $v_{1}$. The flow is a two-layer displacement with a sustained back flow. The leading front velocity is approximated by $\hat{V}_{f}=\hat{V}_{0} V_{2, f}(\chi)$, where $V_{2, f}(\chi)$ comes from the two-layer lubrication model. The back flow undergoes an initial inertial phase before becoming viscous at longer times. We have discussed these flows in Taghavi et al. (2011).

(b) Viscous temporary back flow regime: this regime is defined approximately as flows satisfying $\chi<\chi_{c}, F r=\hat{V}_{0} / \hat{V}_{t}<0.7 \pm 0.1$ and $R e_{t} \cos \beta<25 \pm 5$. In Fig. 17 this regime is marked by $v_{2}$. The trailing front advances initially upstream, against the imposed flow direction, but is eventually arrested and displaced. The transitionary state between this and the exchange flow regime above involves a stationary residual layer, predicted well by $\chi=\chi_{c}$; see Taghavi et al. (2011).

(c) Viscous instantaneous displacement regime: this regime is defined approx- 

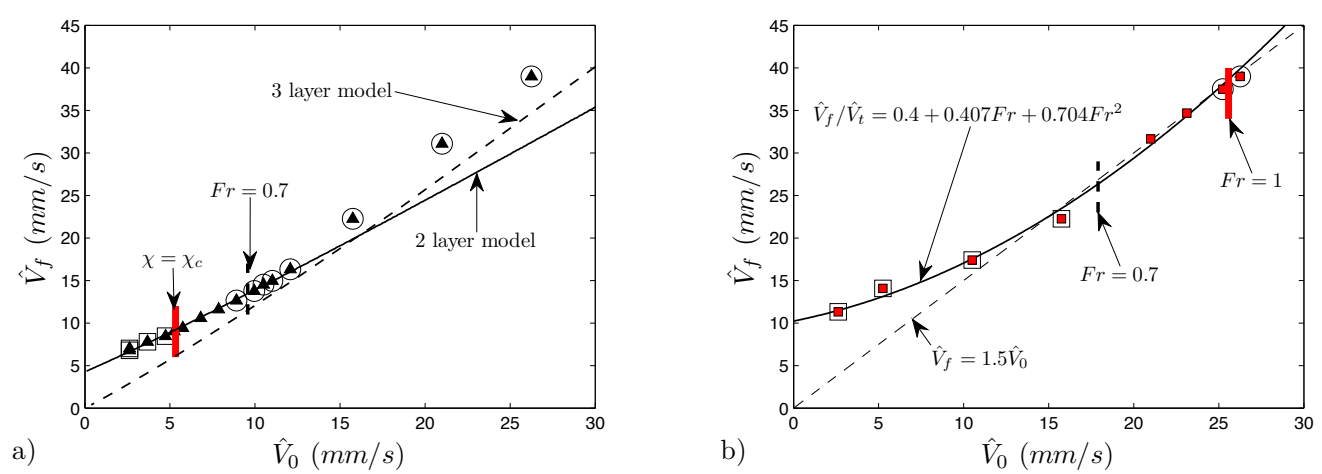

FiguRE 18. a) Front velocity $\hat{V}_{f}$ as a function of mean flow velocity $\hat{V}_{0}$ for a viscous regime displacement $\left(\beta=87^{\circ}, A t=10^{-3}, \nu=1 \mathrm{~mm}^{2} \cdot \mathrm{s}^{-1}, R e_{t} \cos \beta \approx 13\right)$ : sustained back flows and instantaneous displacements are marked by the superposed squares and circles respectively, data points without marks are either temporary back flows or stationary interfaces. The solid line represents the two-layer approximation, $\hat{V}_{f}=\hat{V}_{0} V_{2, f}(\chi)$, and the broken line represents the three-layer approximation, $\hat{V}_{f}=\hat{V}_{0} V_{3, f}(\chi)$. The thick vertical line is at $\chi=\chi_{c}$ and the broken vertical line is at $\hat{V}_{0} / \hat{V}_{t}=0.7$. b) Variation of the front velocity $\hat{V}_{f}$ as a function of $\hat{V}_{0}$ for a sequence of inertial regime displacements, $\left(\beta=83^{\circ}, A t=3.5 \times 10^{-3}, \nu=1\left(\mathrm{~mm}^{2} . \mathrm{s}^{-1}\right.\right.$, $\left.R e_{t} \cos \beta \approx 59\right)$ : sustained back flows and instantaneous displacements are marked by the superposed squares and circles respectively; data points without marks are either temporary back flows or stationary interfaces. The solid line shows the prediction (3.6) and the broken line shows the prediction (3.7). The thick vertical lines are at $F r=\hat{V}_{0} / \hat{V}_{t}=0.7,1$.

imately by $\chi<\chi_{c}, F r=\hat{V}_{0} / \hat{V}_{t}>0.7 \pm 0.1$ and $R e_{t} \cos \beta<25 \pm 5$. In Fig. 17 this regime is marked by $v_{3}$. At smaller values of $\chi$ the front advances predominantly along the channel centre. The front velocity is approximated by $\hat{V}_{f}=\hat{V}_{0} \max \left\{V_{2, f}(\chi), V_{3, f}(\chi)\right\}$, where $V_{3, f}(\chi)$ comes from the three-layer lubrication model. The three-layer model has faster front velocities for $\chi<23$.

(d) Inertial exchange flow dominated regime: this regime is defined as flows for which $F r=\hat{V}_{0} / \hat{V}_{t}<0.7 \pm 0.1$ and $R e_{t} \cos \beta>25 \pm 5$. In Fig. 17 this regime is marked by $i_{1}$. The leading front velocity can be predicted by the empirical model (3.6).

(e) Inertial temporary back flow regime: this regime is found for $0.7 \pm 0.1<$ $F r=\hat{V}_{0} / \hat{V}_{t}<1 \pm 0.1$ and $R e_{t} \cos \beta>25 \pm 5$. In Fig. 17 this regime is marked by $i_{2}$. The front velocity is still predicted by (3.6).

$(f)$ Inertial instantaneous displacement regime: this regime is found for $\mathrm{Fr}=$ $\hat{V}_{0} / \hat{V}_{t}>1 \pm 0.1$ and $R e_{t} \cos \beta>25 \pm 5$. In Fig. 17 this regime is marked by $i_{3}$. The dynamics of the flow is strongly influenced by mixing between the fluids. In this regime (3.7) gives a good approximation for the front velocity.

Figure 18a shows an example of the front velocity prediction for a sequence of purely viscous displacement flows as $\hat{V}_{0}$ is increased and we transition through the regimes $v_{1}$ $v_{3}$. An example of the usage of the predictive models in the inertial regime is shown in Fig. 18b, for a sequence of inertial displacement flows as $\hat{V}_{0}$ is increased and we transition through the inertial regimes, $i_{1}-i_{3}$..

Although we have good agreement with these simple models for the front velocity and the flow regimes, within the range of our numerical experiments, we feel some caution is needed in extending the range of our results. For our inertial regime study we have considered low $A t$ and $\beta \approx 90^{\circ}$ (which has meant that typically $R e_{t} \cos \beta=\lesssim 100$ ). In the range $R e_{t} \cos \beta=\gtrsim 100$, for those flows we have studied, we typically observe that 
coherent vortices are able to cut the channel(s) of pure fluid feeding the front(s) during the displacement. The dynamic of the flow is progressively defined by the mixing between the two fluid layers, rather than by inertial effects present in the bulk flow of at least one layer. Since the (usually narrower) trailing back flow layer is periodically cut by vortices, it also becomes hard to define sustained and temporary back flows in a consistent manner. Thus correctly, our results should be interpreted as applying to weakly inertial regimes.

Finally, we should explain why the pipe displacement flow is laminarised by increasing $\operatorname{Re}\left(\hat{V}_{0}\right)$, but the channel displacement flow evidently is not. In fact, in the channel we can observe some reduction in mixing between the two fluids, but increasing $\hat{V}_{0}$ never completely laminarises the flow. The reason may be associated with the layered structure. The pipe displacements slump to the bottom of the pipe and the interface elongates during the displacement. The plane channel displacement front propagates along the middle of the channel. The elongated interface in the pipe (and upper interface in the channel) are stabilised as the displacement progresses. However, the lower interface in the channel remains mechanically unstable. Potentially it is this buoyancy effect at the lower interface that instigates instability and maintains these flows in the inertial regime.

\section{Inertial effects on plane channel displacements}

We now turn to the consideration of inertial effects via semi-analytical methods. The main tool is a two-layer weighted residual displacement model, in which leading order inertial terms are included in a long-wave lubrication-style model ( $\$ 4.1)$. Unlike the previous two sections where we could make direct comparisons between simplified modeling approaches and (physical or numerical) experiments, here our objective is purely to gain insight. For reasons of simplicity we restrict our attention to the two-layer configuration of see Fig. 14a, and consider only the plane channel geometry.

Extending the pipe flow lubrication-style model to include inertial effects in the same way appears difficult. The single interface configuration is observed in all our pipe flow experiments, where our non-inertial lubrication model under-predicts front velocities as $\hat{V}_{0}$ increases. By studying the effects of including inertia (within the channel geometry) we hope to gain insight into whether inertia could be responsible for this under-prediction.

The two-layer configuration is also observed in our plane channel numerical simulations in the exchange flow dominated regime. In the viscous/laminar dominated regime (at higher $\hat{V}_{0}$ ) the 3-layer approach of $\S 3.4 .1$ may be more appropriate for low At displacements, but we have often observed a diffuse lower interface/layer in these flows, so that the actual flow is neither 2-layer or 3-layer. In any case, some insight is also gained in the effects of inertia on front propagation; see $\S 4.2$ below.

The second area where we apply our analysis is in flow stability ( $§ 4.3)$. The weighted residual approach adopted leads to an extended lubrication model, the stability of which can be analysed. Here we combine a long wavelength linear temporal stability analysis with numerical solution of the evolution equations for given localised initial conditions (a spatio-temporal approach towards convective instability). The main idea here is to gain some predictive insight into the transition to instability-driven mixing of these flows.

\subsection{A weighted residual lubrication model}

We consider a two-dimensional plane channel displacement in which the two fluids are separated by a single-valued interface at $y=h(x, t)$. Assuming a long-thin flow with aspect ratio $\delta$ and adopting the usual scaling arguments, the flow is modeled to $O(\delta)$ by 
the following reduced system of equations:

$$
\begin{aligned}
\delta(1 \pm A t) R e\left[\frac{\partial u}{\partial T}+u \frac{\partial u}{\partial \xi}+V \frac{\partial u}{\partial y}\right] & =-\frac{\partial P}{\partial \xi}+\frac{\partial}{\partial y} \tau_{k, \xi y} \pm \frac{\chi}{2}+O\left(\delta^{2}\right), \\
-\frac{\partial P}{\partial y} \mp \delta \frac{\chi}{2}+O\left(\delta^{2}\right) & =0 \\
\frac{\partial u}{\partial \xi}+\frac{\partial V}{\partial y} & =0 .
\end{aligned}
$$

where the \pm refers to heavy and light fluid layers, respectively. No slip conditions are satisfied at the walls. At the interface both velocity and stress are continuous. The kinematic equation governs evolution of the interface.

The variables $\xi$ and $T$ are rescaled axial length and time, respectively, i.e. $\xi=\delta x, T=$ $\delta t$. We can either interpret $\delta$ as the ratio of $\hat{D}$ to some arbitrary axial length-scale $\hat{L}$, or link $\delta$ back into the physical problem variables. For example, if we adopt the approach of Taghavi et al. (2009), in which it is assumed that the dynamics of spreading of the interface, relative to the mean flow, will be driven by buoyant stresses which have size: $\left|\hat{\rho}_{1}-\hat{\rho}_{2}\right| \hat{g} \sin \beta \hat{D}$ which act via the slope of the interface $\hat{D} / \hat{L}$. The buoyant stresses are balanced by viscous stresses which leads to:

$$
\delta^{-1}=\frac{\hat{L}}{\hat{D}}=\frac{\left|\hat{\rho}_{1}-\hat{\rho}_{2}\right| \hat{g} \sin \beta \hat{D}^{2}}{\hat{\mu} \hat{U}_{0}},
$$

and we may deduce that: $2 \delta R e \sin \beta=F r^{2}$.

The method of analysis of (4.1)-(4.3) stems from the weighted residual approach proposed by Ruyer-Quil \& Manneville (2000) for thin film flows. This has been extended to 2-layer channel flows by Amaouche et al. (2007), whom we largely follow; see also the core-annular flow treatment in Mehidi \& Amatousse (2009). The streamwise velocity components in fluid $k$ are denoted with subscript $k$ and are expanded in powers of $\delta$ :

$$
u_{k}=u_{k}^{(0)}+\delta u_{k}^{(1)}+O\left(\delta^{2}\right) \quad k=1,2
$$

Substituting this expansion into the $x$-momentum equations (4.1) indicates that the leading order solutions, $u_{k}^{(0)}$ are maximum of degree two in $y$ :

$$
u_{k}^{(0)}(\xi, y, T)=A_{k}(\xi, T) y^{2}+B_{k}(\xi, T) y+C_{k}(\xi, T), \quad k=1,2
$$

with the coefficients to be determined. Having fixed the dependency of the leading order streamwise velocity components on $y$, the $x$-momentum equations (4.1) can be integrated with respect to $y$. This simplifies if before the integration, we can multiply the $x$-momentum equations by some suitable weight functions, $g_{k}(\xi, y, T)$ such that unknown terms are eliminated. In particular we define the weight functions in such a way that the averaged equations are no longer dependent on the first-order-terms in the velocity field expansion (4.5). The following 5 conditions are sufficient for this:

$$
\begin{array}{cc}
\int_{0}^{h} g_{1} d y+\int_{h}^{1} g_{2} d y=0, & \left.\frac{\partial g_{1}}{\partial y}\right|_{y=h}-\left.\frac{\partial g_{2}}{\partial y}\right|_{y=h}=0, \\
g_{1}(\xi, h, T)=g_{2}(\xi, h, T), \quad g_{1}(\xi, 0, T)=0, \quad g_{2}(\xi, 1, T)=0,
\end{array}
$$

(see Amaouche et al. (2007) for details). If the $g_{k}$ are chosen as polynomials in $y$, they 
must be at least quadratic:

$$
g_{k}(\xi, y, T)=D_{k}(\xi, T) y^{2}+E_{k}(\xi, T) y+F_{k}(\xi, T)
$$

where $D_{k}, E_{k}$ and $F_{k}$ are functions of $\xi$ and $T$ yet to be known. The five conditions on $g_{k}$ are applied to the six unknowns $D_{k}, E_{k}$ and $F_{k}$, and a sixth condition comes from normalising $D_{1}$. The weight functions $g_{k}$ and the coefficients $A_{k}, B_{k}$ and $C_{k}(k=1,2)$ are given in Alba et al. (2011). The leading order depthwise velocity component is recovered from the continuity equation (4.3). Integrating the $x$-momentum equations (4.1) with respect to $y$ over each fluid layer and summing them, results after some algebra in:

$$
\begin{aligned}
0= & \int_{0}^{h}\left[\delta(1+A t) \operatorname{Re}\left(\frac{\partial u_{1}^{(0)}}{\partial t}+u_{1}^{(0)} \frac{\partial u_{1}^{(0)}}{\partial x}+V_{1}^{(0)} \frac{\partial u_{1}^{(0)}}{\partial y}\right)-\frac{\partial^{2} u_{1}^{(0)}}{\partial y^{2}}\right] g_{1} d y+ \\
& \int_{h}^{1}\left[\delta(1-A t) \operatorname{Re}\left(\frac{\partial u_{2}^{(0)}}{\partial t}+u_{2}^{(0)} \frac{\partial u_{2}^{(0)}}{\partial x}+V_{2}^{(0)} \frac{\partial u_{2}^{(0)}}{\partial y}\right)-\frac{\partial^{2} u_{2}^{(0)}}{\partial y^{2}}-\frac{\partial h}{\partial x}+\chi\right] g_{2} d y .
\end{aligned}
$$

We substitute the velocity components and the weight functions, expressed in terms of their coefficients, into (4.8) and carry out the integrations. After considerable algebra (performed symbolically with MAPLE ${ }^{T M}$, version 12) it is found that each term can be expressed in terms of the flux $q$ through the lower layer and the interface height $h$. We are left with the kinematic condition and (4.8), which governs evolution of $q$. The coupled system is:

$$
\begin{aligned}
\frac{\partial h}{\partial T}+\frac{\partial q}{\partial \xi} & =0 \\
R_{1} \frac{\partial h}{\partial T}+R_{2} \frac{\partial h}{\partial \xi}+R_{3} \frac{\partial q}{\partial T}+R_{4} \frac{\partial q}{\partial \xi}+R_{5} & =0
\end{aligned}
$$

where $R_{1}, R_{2}, \ldots R_{5}$ are explicit algebraic functions of $q, h, \delta R e$ and $\chi$; see appendix $\S \mathrm{B}$.

\subsection{Inertial effects on front shape and speed}

To study inertial effects on the displacement front we integrate (4.9) \& (4.10) numerically. The kinematic condition (4.9) is discretized in conservative form, second order in space and first order explicitly in time. It is integrated using a Van Leer flux limiter scheme; see Yee et al. (1985). For (4.10) the same flux limiter scheme has been used. However, (4.9) $\&$ (4.10) are solved sequentially and we have used updated values for $h$ in the solution of (4.10), making the scheme semi-implicit. We have benchmarked our computational method by comparison with results from the lubrication model in Taghavi et al. (2009), giving an acceptable comparison.

Typical evolution of the interface globally mimics that of the lubrication model in Taghavi et al. (2009). After initial transients the interface either advances fully downstream (small $\chi$ ) or can have both a downstream moving front and an second front moving upstream (large $\chi$ ). Here we are mostly interested in the inertial correction to the downstream front in the laminar/viscous dominated range (small $\chi$ ). Figure 19a shows examples of the interface shapes $h(\xi, T)$ at $T=10$ for $\delta R e=0.01,5,50$, all with $\chi=0$. For $\delta R e \ll 1$ the interface shape is indistinguishable from that of the lubrication model. As inertial effects $\delta R e$ become significant we can see marked changes in the interface shape. In general the transition from stretched interface to front is smoothed out and the front height is reduced. The frontal region for the lubrication model is not a kinematic shock, but is a region in which the diffusive effects of gravitational spreading remain significant. The addition of inertia appears to extend this region axially along the 

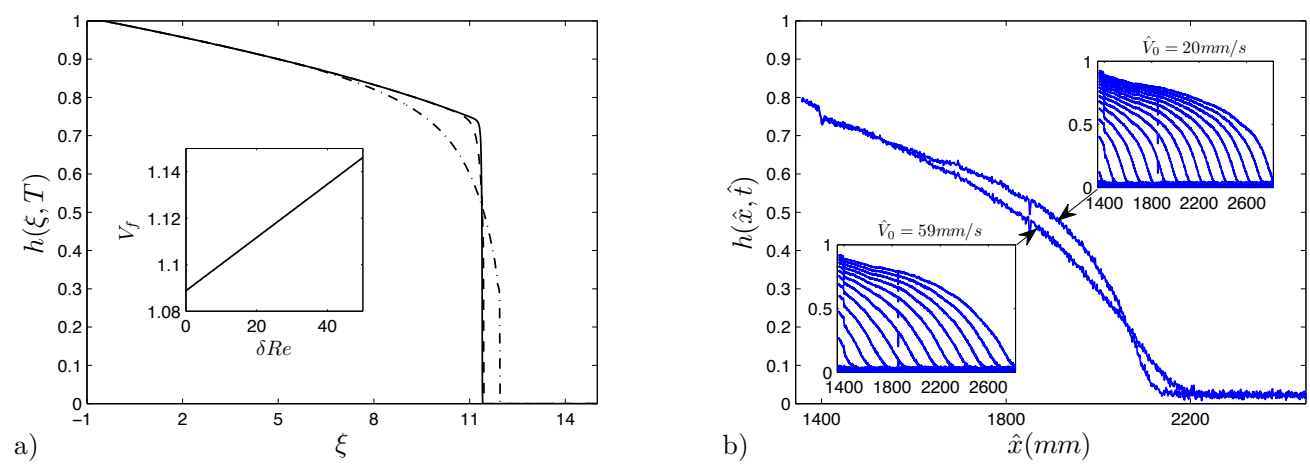

FiguRE 19. Front velocity and shape influences at $\chi=0$ : comparison of interface shapes $h(\xi, T)$ at $T=10$ for $\delta R e=0.01$ (solid line), 5, 50 (broken lines); the inset shows the variation of downstream front velocity, $V_{f}$ versus $\delta R e$. b) Two experimental profiles of normalized $h(\hat{x}, \hat{t})$ for $\beta=87^{\circ}, A t=10^{-3}, \hat{\nu}=1\left(\mathrm{~mm}^{2} \cdot \mathrm{s}^{-1}\right)$ : the insets indicate interfaces for $\hat{t}=60,65, . ., 125,130(\mathrm{~s})$ : left down $\hat{V}_{0}=20\left(\mathrm{~mm} . \mathrm{s}^{-1}\right),(\chi=21, \delta R e=0.84)$; right up $\hat{V}_{0}=59\left(\mathrm{~mm} \cdot \mathrm{s}^{-1}\right),(\chi=6, \delta R e=9.39)$.

channel. The reduction in front height leads to a consequent increase in the downstream front speed, which is calculated and shown in Fig. 19a (inset), again for $\chi=0$ (meaning large $\hat{V}_{0}$ ). We observe a near linear increase with $\delta R e$. Similar effects are observed for other values of $\chi$.

Considering the potential for this effect to explain the discrepancy in front speeds measured in the pipe flow displacements, it appears to be a plausible explanation. The scale of increase in front speed is significant if we consider that $V_{f}=\hat{V}_{f} / \hat{V}_{0}>1$ for any displacement in this laminar/viscous regime and we have seen that front velocities are also bounded by the zero At limit of the 3-layer flow, i.e. $V_{f}<1.5$. In the case of the pipe flow there is an analogous zero $A t$ limit in the interfacial velocity: $V_{f}<4 / 3 . \dagger$ Thus, an increase in $V_{f}$ of size $\sim 0.05$ over the experimental range of $\delta R e$ would be significant for the pipe flow.

If we return to the data from our pipe flow experiments in the laminarised/viscous regime, although at leading order the increase in $\hat{V}_{f}$ with $\hat{V}_{0}$ is approximately linear, close inspection reveals that $\hat{V}_{f}$ increases with $\hat{V}_{0}$ slightly more than linearly in all experimental series, as would be the case with an inertial correction. Other parametric changes in the slope of the curve $\hat{V}_{f}$ vs $\hat{V}_{0}$ are that: (i) the slope increases as pipe inclination becomes steeper; (ii) the slope increases as At decreases. Both these qualitative trends are in the same direction as predictions from our inertial two-layer model.

Lastly, we examine changes in front shape at similar $\chi$ and $\delta R e$ observed in the pipe flow experiments and in our model. Note that in an experimental sequence, as we increase $\hat{V}_{0}$ we both decrease $\chi$ and increase $\delta R e$. In our model, the decrease in $\chi$ tends to decrease the front velocity and increase the front height, an effect which competes against that of $\delta R e$. The insets of Fig. 19b show the evolution of the normalised concentration measured in 2 pipe flow experiments (with $\beta=87^{\circ}, A t=10^{-3}, \hat{\nu}=1\left(\mathrm{~mm}^{2} \cdot \mathrm{s}^{-1}\right)$ ), for different $\chi$ and $\delta R e$. In the main figure we overlay two interface profiles at a later time and we observe that the frontal region enlarges under the effect of increasing the imposed flow while the front height decreases.

$\dagger$ Note that for the pipe flow this limit is not equal to the scaled centreline velocity, but to the average of the velocity in the spanwise direction. 


\subsection{Flow stability}

The interface typically consists of propagating frontal regions connected by interfaces that essentially stretch at long times. As the interface elongates in these connecting regions we might reasonably analyze the flow stability via perturbing the flow about a constant uniform interface height $h_{s}$, with corresponding steady flux $q_{s}$. For different $\chi$ and $\delta R e$ the front heights change so that different ranges of $h$ are likely to be found in practice. For example, at large $\chi$ where we have a backflow, we expect only the intermediate values of $h$ to be stretched out between the fronts at long times. For small $\chi$ the entire interface has positive velocity and will be stretched over a wider range of larger heights $h$.

Two methods of analysis are adopted. Firstly we consider a linear temporal stability analysis in the long wave limit, which can be performed analytically. Secondly, we consider a numerical approach, imposing a localised finite initial perturbation on the interface and observing whether it grows or decays. This might be termed a spatiotemporal approach.

\subsubsection{Long-wave temporal linear stability analysis}

Here we take a classical modal approach, perturbing (4.9) and (4.10) about a uniform steady solution $\left(h_{s}, q_{s}\right)$. The steady state satisfies the following relation:

$$
q_{s}=h_{s}^{2}\left(3-2 h_{s}\right)+\frac{\chi h_{s}^{3}\left(1-h_{s}\right)^{3}}{3} .
$$

We suppose a linear perturbation: $h=h_{s}+h^{\prime}, q=q_{s}+q^{\prime}$, substitute into (4.9) and (4.10), and retain only linear terms. We now assume a modal form for the linear perturbations, periodic in $\xi$, so that:

$$
h^{\prime}=\bar{h} \mathrm{e}^{\mathrm{i} \alpha \xi+\sigma T}, \quad q^{\prime}=\bar{q} \mathrm{e}^{\mathrm{i} \alpha \xi+\sigma T},
$$

where $\bar{h}$ and $\bar{q}$ are constants. Substituting (4.12) into the linear stability equations leads to the following dispersion relation, quadratic in $\sigma$.

$$
\left|\begin{array}{cc}
\sigma & i \alpha \\
R_{s, 1} \sigma+R_{s, 2} i \alpha+\frac{\partial R_{s, 5}}{\partial h} & R_{s, 3} \sigma+R_{s, 4} i \alpha+\frac{\partial R_{s, 5}}{\partial q}
\end{array}\right|=0
$$

Here the coefficients $R_{s, k}$ are simply the $R_{k}$ from appendix $\S \mathrm{B}$, evaluated at $\left(h_{s}, q_{s}\right)$. If the real part of $\sigma$ is positive, the flow is linearly unstable.

Although we can find $\sigma$ for any wave number $\alpha$, for all values we have tested the sign of the real part of $\sigma$ is determined by the long wavelength limit $\alpha \rightarrow 0$. This limit is evaluated by expanding the eigenvalue around $\alpha=0$

$$
\sigma=\sigma_{0}+\alpha \sigma_{1}+\alpha^{2} \sigma_{2}+\ldots
$$

We find that $\sigma_{0}=0, \sigma_{1}$ is imaginary and stability is governed by the sign of $\sigma_{2}$. Marginal stability curves are obtained by putting $\sigma_{2}=0$ which leads to:

$$
\delta R e=\frac{140}{\chi\left[3\left(2 h_{s}-1\right)\left(33 h_{s}^{2}-33 h_{s}+2\right)-\chi h_{s}^{2}\left(1-h_{s}\right)\left(73 h_{s}^{3}-146 h_{s}^{2}+92 h_{s}-19\right)\right]}
$$

The marginal stability curves are plotted in Fig. 20a, in the positive quadrant of the $(\chi, \delta R e)$-plane, for different values of $h_{s}$. In this figure long-wave instability is found for large $(\chi, \delta R e)$, exceeding the plotted curves. We observe that each curve asymptotes to $\delta R e=\infty$ at a critical value of $\chi$, that depends on $h_{s}$. These critical values, say $\chi_{s}\left(h_{s}\right)$ are easily calculated:

$$
\chi_{s}\left(h_{s}\right)=\max \left\{0, \frac{3\left(2 h_{s}-1\right)\left(33 h_{s}^{2}-33 h_{s}+2\right)}{h_{s}^{2}\left(1-h_{s}\right)\left(73 h_{s}^{3}-146 h_{s}^{2}+92 h_{s}-19\right)}\right\} .
$$



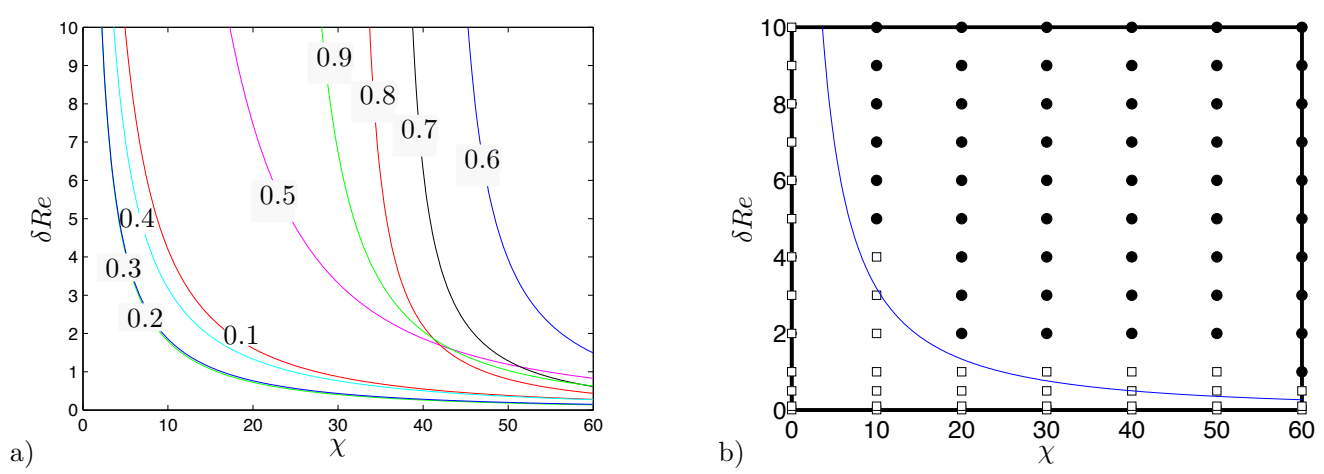

Figure 20. a) Marginal stability curves for the long-wave limit, from (4.15), for the indicated values of $h_{s}$. b) Stability diagram indicating stable flows $(\square)$ and unstable flows $(\bullet)$. Line indicate the neutral curve for the long wave length limit for the interface initially located at $h_{s}=0.4$.

For each value of $h_{s}$, it is necessary for $\chi>\chi_{s}\left(h_{s}\right)$ in order to have instability, and when this condition is satisfied, then the critical $\delta R e$ is found from (4.15).

When we are far from the initial transients and when the displacing front has become fully developed, we can assume that at each streamwise location, the interface height is at an approximately constant value with some slight variation in the $\xi$ direction. The linear stability analysis of the weakly-inertial model (Fig. 20a) shows that the flow can be either stable or unstable at a given $h$, depending on the values of $\chi$ and $\delta R e$. For $0.45<h<1$ Fig. 20a suggests that the flow is least stable for $h \approx 0.5$. Potentially, one could use a single marginal stability curve (e.g. for $h_{s} \approx 0.5$ as an approximate practical criterion in deciding whether the displacement flow is stable or not.

\subsubsection{Spatio-temporal stability}

To complement the linear analysis we also analyse the growth of instabilities numerically. For this we use the full system (4.9) \& (4.10) and solve an initial value problem. For the initial values we fix a constant $h_{s}$ (hence also $q_{s}$ ) and superimpose a localised interfacial disturbance on the initial condition, i.e. our initial $h$ takes the form:

$$
h(\xi, 0)= \begin{cases}h_{s}, & \xi \notin[0,2], \\ h_{s}+A \sin \pi \xi, & \xi \in[0,2]\end{cases}
$$

Typically we take the amplitude $A=0.05$. We now track the response of the system (4.9) \& (4.10) to this forcing, to see if the interface amplitude grows or decays in time and space. We do this via analysis of a spatiotemporal plot of the interface height. The unperturbed values are a steady state of the system (4.9) \& (4.10). The interface perturbation is typically advected dispersively downstream, but may either decay or grow. Two examples of the spatiotemporal stability analysis are shown in Fig. 21a,b for $\chi=65 \& \delta R e=0.24$ and $\chi=35 \& \delta R e=8.98$ respectively. The former shows a case where the perturbation decays with time and space while the latter illustrates growth in the amplitude.

This numerical approach allows us to study nonlinear perturbations and to gain insight into convective aspects of the instability, which are evident in our experiments (physical and numerical). As with any initial value approach there are disadvantages in having to select a particular initial condition. To develop qualitative understanding we have computed the interface evolution for $h_{s}=0.1,0.2, \ldots, 0.8,0.9$, over a wide range of 



FigURE 21. Examples of the spatiotemporal evolution of the interface, $h-h_{s}$ (illustrated by contours of intensity) for interfaces initially located at $h_{s}=0.5$ : a) $\chi=65$ and $\delta R e=0.24$ and b) $\chi=35$ and $\delta R e=8.98$. The inset depicts two sample interfaces, $h(\xi, T)$, at $T=0$ (broken line) and $T=5$ (solid line).

$(\chi, \delta R e)$. According to the spatiotemporal plot we categorize each point as either unstable or stable. As an example, Fig. 20b shows the stability map obtained for a range of $\delta R e$ and $\chi$ at $h_{s}=0.4$, based on this perturbation technique. We also superimpose the marginal stability curve from the long-wave temporal linear stability analysis. It is interesting to note the relatively good agreement between the long-wave temporal linear stability analysis and the spatio-temporal analysis, in the sense that all unstable computations lie above the linear stability criterion. The direct implication of this agreement is that the flow is convectively unstable.

Figure 20b clarifies some behaviors of the flow. Consider any point in the stable zone: moving upwards or moving to the right both destabilize the flow. Moving upwards parallel to the $y$-axis is equivalent to increasing the ratio of the inertial (i.e $\delta R e$ ) to buoyant/viscous forces (since $\chi$ is kept constant). In this case the flow becomes unstable since the inertial forces exceed an instability threshold. Moving to the right parallel to the $x$-axis is equivalent to decreasing viscous forces in the flow, relative to buoyancy forces, which will eventually trigger instability. In the same context, lines $\chi=0$ and $\delta R e=0$ are always stable: $\chi \rightarrow 0$ implies that viscous forces completely dominate the buoyancy; $\delta R e=0$ implies no inertia (i.e. the viscous lubrication model is recovered).

If we consider the denominator of the expression on the right-hand side of (4.15) we observe a term that is linear in $\chi$ and one that is quadratic in $\chi$. The coefficient of the linear term vanishes at $h_{s}=0.5$ whereas the coefficient of the quadratic term vanishes at $h_{s}=0,1$. Thus, we can interpret (4.15), as $h_{s}$ varies, as giving a nonlinear interpolation between conditions of form $\delta R e \chi=$ constant and $\delta R e \chi^{2}=$ constant. The former of these translates straightforwardly into an instability criterion of the form

$$
R e \cot \beta>\text { constant, }
$$

whereas the latter is of the form

$$
\frac{R e_{t} \cos \beta}{\sqrt{\sin \beta}}>\text { constant. }
$$

The former is a typical shear flow instability criterion, ignoring buoyancy, but leads to infinite $R e$ for strictly horizontal channels. This is unrealistic, compared to an OrrSommerfeld type approach, but of course here we have strictly an averaged long-wavelength 
limit. The second condition above is of the same form as that determined empirically and marks the transition between inertial and viscous exchange flows, e.g. see $\S 3.2$. For $h_{s}=0.5$ and considering $\sqrt{\sin \beta} \approx 1$, this relation predicts the transition at $R_{t} \cos \beta=$ $27.32 \ldots$, which is interestingly close to what we computationally obtain (i.e. $R e_{t} \cos \beta=$ $25 \pm 5$ ). Although both effects are expected to be present in any instability, we feel further work is needed to better understand the transition to fully mixed displacement flows.

\section{Summary}

We have presented comprehensive results on miscible displacement flows at low $A t$ in near-horizontal ducts, with iso-viscous Newtonian fluids. Although the flow is controlled by 3 parameters, $(\operatorname{Re}, F r, \beta)$, we have been able to categorize the types of observed flows efficiently in the $(F r, R e \cos \beta / F r)$-plane. In both pipe and plane channel geometries we are able to identify 5-6 different regimes, observed at long times. These are depicted in Fig. 8 \& Fig. 17 for pipe and plane channel geometries respectively.

The main advance with respect to our previous work in Taghavi et al. $(2010,2011)$ is that the classifications previously developed were (largely) qualitative and phenomenological, based on a description of the displacement fronts. Significantly now each of the flow regimes in Fig. 8 \& Fig. 17 is identified quantitatively, i.e. a leading order approximation to the regime boundaries is given. In addition, we have identified each regime as either inertial or viscous. Finally, within each flow regime we have been able to offer a leading order quantitative approximation of the leading front velocity. The leading front velocity has high practical significance as it gives directly a measure of the displacement efficiency, i.e. $V_{f}^{-1}$ indicates the efficiency; see Taghavi et al. (2009). These are the key contributions.

The above results pertain to both geometries considered. It is satisfying that the semianalytical methods we have used (lubrication/thin-film models for the viscous regimes and dimensional analysis for inertial exchange flows) have been able to capture the main trends from our experiments (real in the case of the pipe, numerical in the channel). This combination of methodologies used gives us higher confidence in the validity and robustness of our characterisation. More detail of the experimental results, computations and analysis can be found in Taghavi (2011).

In the final part of our study we have begun to consider how weak inertial effects may modify our viscous theory, by using a weighted residual-type approach. This model shows that inertial effects lead to a modification of the front velocity prediction that is of the order of the discrepancy with our experimental results and that we see qualitatively similar changes in front shape. We have analysed the long-wave temporal linear stability of a two layer flow using this model and have compared these results with a numerical spatio-temporal stability analysis of the same model. The predictions arising from numerical solution of the non-linear equations for the weakly inertial displacement flow are in good agreement with the analytical temporal linear stability results. This confirms the convective nature of the instabilities for this flow. This said, the stability study is quite preliminary and needs development.

\section{Acknowledgements:}

This research has been carried out at the University of British Columbia, supported financially by NSERC and Schlumberger through CRD project 354717-07. 


\section{Appendix A. Three-layer lubrication model}

The flux functions $q_{L}\left(y_{i}, h, y_{i, \xi}, h_{\xi}\right)$ and $q\left(y_{i}, h, y_{i, \xi}, h_{\xi}\right)$ are defined as follows.

$$
\begin{aligned}
q_{L}= & y_{i}^{2}\left(3-2 y_{i}\right)+\frac{\left[\chi-y_{i, \xi}\right] y_{i}^{2} h}{6}\left[h^{2}\left(3-2 y_{i}\right)+3\left(1-y_{i}\right)^{2}\left(1+2 y_{i}-2 h\right)\right] \\
& \frac{y_{i}^{2} h_{\xi}}{6}\left[h y_{i}\left(12-12 h-15 y_{i}+2 h^{2}+6 y_{i} h+6 y_{i}^{2}\right)-3 h(1-h)^{2}-2 y_{i}\left(1-y_{i}\right)^{3}\right] \\
q= & h^{2}(3-2 h)+6 y_{i} h\left[1-y_{i}-h\right]+ \\
& {\left[\chi-y_{i, \xi}\right]\left[\frac{h^{3}(1-h)^{3}}{3}+y_{i} h^{2}\left(1-y_{i}-h\right)\left([1-h][1-2 h]-3 y_{i}\left[1-y_{i}-h\right]\right)\right]+} \\
& \frac{h_{\xi}}{6}\left[-2 h^{3}(1-h)^{3}+6 h^{2} y_{i}(1-h)^{2}(2 h-1)+3 h y_{i}^{2}\left(1-y_{i}\right)^{2}\left(2 y_{i}-1\right)\right]+ \\
& \frac{y_{i}^{2} h^{2} h_{\xi}}{6}\left[24\left(1-y_{i}\right)^{2}+30(1-h)^{2}+38 y_{i} h+3 h-24\right]
\end{aligned}
$$

\section{Appendix B. The coefficients $R_{1} \ldots R_{5}$}

$$
\begin{gathered}
R_{1}=\frac{\left(2 h^{4}-4 h^{3}+3 h^{2}-2 h q+q\right) \delta R e}{10(h-1)\left(2 h^{2}-h-1\right)} \\
R_{2}=\frac{\delta R e\left(54 h^{6}-90 h^{5}+9 h^{4}-54 h^{3}+q h\left(-144 h^{4}+360 h^{3}-108 h^{2}+72 h-18\right)\right)}{840\left(2 h^{3}-3 h^{2}+1\right)(h-1) h} \\
+\frac{\delta R e q^{2}\left(-270 h^{2}+270 h-81\right)+h^{4}\left(280 h^{4}-1120 h^{3}+1680 h^{2}-1120 h+280\right)}{840\left(2 h^{3}-3 h^{2}+1\right)(h-1) h} \\
R_{3}=\frac{\delta R e h}{10(h-1)(2 h+1)} \\
R_{4}=\frac{\left(64 h^{4}-128 h^{3}+43 h^{2}-6 h+54 h q-27 q\right) \delta R e}{280(h-1)\left(2 h^{2}-h-1\right)} \\
R_{5}=\frac{\chi\left(h^{6}-3 h^{5}+3 h^{4}-h^{3}\right)+6 h^{3}-9 h^{2}+3 q}{3(-2 h-1)(h-1)^{2}}
\end{gathered}
$$

\section{REFERENCES}

Alba, K., Laure, P. \& Khayat, R.E. 2011 Transient two-layer thin-film flow inside a channel. Phys. Rev. E 84, 026320.

Amaouche, M., Mehidi, N. \& Amatousse, N. 2007 Linear stability of a two-layer film flow down an inclined channel: A second-order weighted residual approach. Phys. Fluids 19, 084106.

Baird, M.H.I., Aravamudan, K., Rao, N.V. Rama, Chadam, J. \& Peirce, A.P. 1992 Unsteady axial mixing by natural convection in vertical column. AIChE J. 38, 1825 .

Beckett, F., Mader, H.M., Phillips, J.C., Rust, A. \& Witham, F. 2011 An experimental study of low Reynolds number exchange flow of two Newtonian fluids in a vertical pipe. Preprint submitted to . J. Fluid Mech. .

Benjamin, T.J. 1968 Gravity currents and related phenomena. J. Fluid. Mech. 31 (209).

Birman, V.K., Battandier, B.A., Meiburg, E. \& Linden, P.F. 2007 Lock-exchange flows in sloping channels. J. Fluid Mech. 577, 53-77. 
Birman, V.K., Martin, J.E., Meiburg, E. \& Linden, P.F. 2005 The non- Boussinesq lockexchange problem. Part 2. High- resolution simulations. J. Fluid Mech. 537, 125-144.

Charru, F. \& Hinch, E.J. 2000 Phase diagram of interfacial instabilities in a two-layer Couette flow and mechanism of the long wave instability. J. Fluid Mech. 414, 195223.

Chen, C.-Y. \& Meiburg, E. 1996 Miscible displacements in capillary tubes. part 2. numerical simulations. J. Fluid Mech. 326, 57-90.

Debacq, M., Fanguet, V., Hulin, J.P., Salin, D. \& Perrin, B. 2001 Self similar concentration profiles in buoyant mixing of miscible fluids in a vertical tube. Phys. Fluids 13, 3097.

Debacq, M., Hulin, J.P., Salin, D., Perrin, B. \& Hinch, E.J. 2003 Buoyant mixing of miscible fluids of varying viscosities in vertical tube. Phys Fluids 15, 3846.

Didden, N. \& MAXworthy, T. 1982 The viscous spreading of plane and axisymmetric gravity currents. J. Fluid Mech. 121, 27-42.

D'Olce, M. 2008 Instabilités de cisaillement dans lécoulement concentrique de deux fluides miscibles. PhD thesis, These de l'Universite Pierre et Marie Curie, Orsay, France.

D'Olce, M., Martin, J., Rakotomalala, N. \& Salin, D. 2008 Pearl and mushroom instability patterns in two miscible fluids core annular flows. Phys. Fluids 20, 024104.

D’Olce, M., Martin, J., Rakotomalala, N., Salin, D. \& Talon, L. 2009 Convective/absolute instability in miscible core-annular flow. part 1. experiments. J. Fluid Mech. 618, 305-322.

Ern, P., Charru, F. \& Luchini, P. 2003 Stability analysis of a shear flow with strongly stratified viscosity. J. Fluid Mech. 496, 295312.

Govindarajan, R. 2004 Effect of miscibility on the linear instability of two-fluid channel flow. Int. J. Multiphase Flow 30, 1177-1192.

Goyal, N. \& Meiburg, E. 2006 Miscible displacements in Hele-Shaw cells: two-dimensional base states and their linear stability. J. Fluid Mech. 558, 329355.

Goyal, N., Pichler, H. \& Meiburg, E. 2007 Variable density, miscible displacements in a vertical Hele-Shaw cell: linear stability. J. Fluid Mech. 584, 357372.

Hallez, Y. \& Magnaudet, J. 2008 Effects of channel geometry on buoyancy-driven mixing. Phys. Fluids 20, 053306.

Hinch, E.J. 1984 A note on the mechanism of the instability at the interface between two shearing fluids. J. Fluid Mech. 114, 463465.

Hormozi, S., Wielage-Burchard, K. \& FrigaArd, I.A. 2011 Entry, start up and stability effects in visco-plastically lubricated pipe flows. J. Fluid Mech. 673, 432-467.

Hoult, D.P 1972 Oil spreading in the sea. Annu. Rev. Fluid Mech. 4, 341-\&.

Huppert, H. E. \& Hallworth, M. A. 2007 Bi-directional flows in constrained systems. J. Fluid Mech. 578, 95-112.

Joseph, D. D. \& Renardy, Y. Y. 1993 Fundamentals of Two-Fluid Dynamics. Part 2: Lubricated transport, drops and miscible liquids, , vol. 4. Springer, Interdisciplinary Applied Mathematics Series.

Lajeunesse, E., Martin, J., Rakotomalala, N., Salin, D. \& Yortsos, Y. 1999 Miscible displacement in a Hele Shaw cell at high rates. J. Fluid Mech. 398, 299-319.

Lajeunesse, E., Martin, J And, Rakotomalala N. \& Salin, D. 1997 3d instability of miscible displacements in a Hele-Shaw cell. Phys. Rev. Lett. 79, 5254-5257.

Mehidi, N. \& Amatousse, N. 2009 Modélisation dun écoulement coaxial en conduite circulaire de deux fluides visqueux. C.R. Mecanique 337, 112-118.

Petitjeans, P. \& Maxworthy, T. 1996 Miscible displacements in capillary tubes. part 1. experiments. J. Fluid Mech. 326, 37-56.

Rakotomalala, N., Salin, D. \& Watzky, P. 1997 Miscible displacement between two parallel plates: BGK lattice gas simulations. J. Fluid Mech. 338, 277-297.

Ranganathan, T. \& Govindarajan, R. 2001 Stabilization and destabilization of channel flow by location of viscosity-stratified fluid layer. Phys. Fluids 13, 1-3.

Ruyer-Quil, C. \& Manneville, P. 2000 Improved modeling of flows down inclined planes. Eur. Phys. J. B 15, 357-369.

Sahu, K.C., Ding, H., Valluri, P. \& Matar, O.K. $2009 a$ Linear stability analysis and numerical simulation of miscible two-layer channel flow. Phys. Fluids 21, 042104. 
Sahu, K.C., Ding, H., Valluri, P. \& Matar, O.K. $2009 b$ Pressure-driven miscible two-fluid channel flow with density gradients. Phys. Fluids 21, 043603.

Selvam, B., Merk, S., Govindarajan, R. \& Meiburg, E. 2007 Stability of miscible coreannular flow with viscosity stratification. J. Fluid Mech. 492, 2349.

Selvam, B., Talon, L., Leshafft, L. \& Meiburg, E. 2009 Convective/absolute instability in miscible coreannular flow. Part 2. Numerical simulations and nonlinear global modes. J. Fluid Mech. 618, 323348.

Seon, T., Hulin, J.-P., SAlin, D., Perrin, B. \& Hinch, E.J. 2004 Buoyant mixing of miscible fluids in tilted tubes. Phys. Fluids 16, L103-L106.

Seon, T., Hulin, J.-P., Salin, D., Perrin, B. \& Hinch, E.J. 2005 Buoyancy driven miscible front dynamics in tilted tubes. Phys. Fluids 17, 031702.

Seon, T., Hulin, J.-P., Salin, D., Perrin, B. \& Hinch, E.J. 2006 Laser-induced fluorescence measurements of buoyancy driven mixing in tilted tubes. Phys. Fluids 18, 041701.

Seon, T., Znaien, J., Salin, D., Hulin, J.-P., Hinch, E.J. \& Perrin, B. $2007 a$ Front dynamics and macroscopic diffusion in buoyant mixing in a tilted tube. Phys. Fluids 19, 125105.

Seon, T., Znaien, J., Salin, D., Hulin, J.-P., Hinch, E.J. \& Perrin, B. $2007 b$ Transient buoyancy-driven front dynamics in nearly horizontal tubes. Phys. Fluids 19, 123603.

Shin, J.O., DAlziel, S.B. \& Linden, P.F. 2004 Gravity currents produced by lock exchange. J. Fluid. Mech. 521, 1-34.

Simpson, J.E. 1997 Gravity Currents in the Environment and the Laboratory, 2nd edn. Cambridge University Press, Cambidge.

Stevenson, D.S. \& Blake, S. 1998 Modelling the dynamics and thermodynamics of volcanic degassing. Bull. Volcanol. 38 (4), 307-317.

TAghavi, S.M. 2011 From displacement to mixing in a slightly inclined duct. PhD thesis, University of British Columbia, Vancouver, Canada.

Taghavi, S.M., Seon, T., Martinez, D.M. \& Frigaard, I.A. 2009 Buoyancy-dominated displacement flows in near-horizontal channels: the viscous limit. J. Fluid. Mech. 639, $1-35$.

Taghavi, S.M., Seon, T., Martinez, D.M. \& FrigaArd, I.A. 2010 Influence of an imposed flow on the stability of a gravity current in a near horizontal duct. Phys. Fluids 22, 031702.

Taghavi, S.M., Seon, T., Wielage-Burchard, K., Martinez, D.M. \& Frigaard, I.A. 2011 Stationary residual layers in buoyant newtonian displacement flows. Phys. Fluids 23, 044105.

YAnG, Z. \& Yortsos, Y. C. 1997 Asymptotic solutions of miscible displacements in geometries of large aspect ratio. Phys. Fluids 9, 286-298.

Yee, H.C., Warming, R.F. \& Harten, A. 1985 Implicit Total Variation Diminishing (TVD) Schemes for Steady-State Calculations. J. Comput. Phys. 57, 327-360.

YIH, C.-S. 1967 Instability due to viscosity stratification. J. Fluid Mech. 27, 337352. 